\title{
Flat Connections and Geometric Quantization
}

\author{
N. J. Hitchin $\star$ \\ Mathematical Institute, 24-29 St. Giles, Oxford OX1 3LB, United Kingdom
}

\begin{abstract}
Using the space of holomorphic symmetric tensors on the moduli space of stable bundles over a Riemann surface we construct a projectively flat connection on a vector bundle over Teichmüller space. The fibre of the vector bundle consists of the global sections of a power of the determinant bundle on the moduli space. Both Dolbeault and Čech techniques are used.
\end{abstract}

\section{Introduction}

The new invariants of 3-manifolds introduced by Witten [21] can be approached by defining a vector space $V$ canonically associated to a closed surface $\Sigma$, a Lie group $G$, and an integer $k$. These spaces are to be thought of as analogues of cohomology groups, though satisfying different functorial properties [17]. To define cohomology groups one usually requires a choice of auxiliary structure - a triangulation, Čech covering, differentiable structure, or Riemannian metric - and one needs to prove that the resulting space is independent, in a suitable sense, of that choice. The same is true of the vector spaces required for Witten's theory, and the aim of this paper is to prove that independence for the case $G=S U(m)$.

The underlying idea behind the vector space $V$ is that of the geometric quantization of a symplectic manifold $M$. Given the group $G$, we consider the space of irreducible representations of the fundamental group $\pi_{1}(\Sigma)$ into $G$ :

$$
\operatorname{Hom}\left(\pi_{1}(\Sigma), G\right)^{\mathrm{irr}} / G,
$$

which is in a canonical way a symplectic manifold $M$. Multiplying the canonical symplectic form by the level $k$ gives it a different symplectic structure. These symplectic manifolds are clearly canonically associated to the surface $\Sigma$. To quantize them, in the Kostant-Kirillov-Souriau sense, requires a choice of polarization and one then needs to prove that the space is independent of that

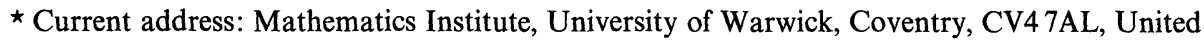
Kingdom 
choice. One type of polarization is a Kähler polarization - we choose a complex structure on $M$ to make it into a Kähler manifold. This is an appropriate thing to do in the present context since a choice of conformal structure on $\Sigma$ induces a natural complex structure on $M$ which defines a Kähler polarization. As a complex manifold $M$ becomes, due to the theorem of Narasimhan and Seshadri, the moduli space of stable holomorphic vector bundles of rank $m$ over the Riemann surface $\Sigma$.

For a Kähler polarization, the vector space $V$ one takes for the geometric quantization is the space of global holomorphic sections of a holomorphic line bundle $L$ which has a hermitian metric whose curvature form is the symplectic form $\omega$. It is a general feature of quantization that only the projective space $\mathbb{P}(V)$ is canonically defined - in the presence of group actions on $M$ one tends to get projective representations or representations of central extensions. This means that what we look for is a canonical way of identifying the projective spaces for two different conformal structures on the surface $\Sigma$. This may be regarded as parallel translation for a flat connection on the bundle of projective spaces $\mathbb{P}(V)$ over Teichmüller space. Given a diffeomorphism $f$ of $\Sigma$, one may then compare parallel translation between the points $x$ and $f(x)$ in Teichmüller space with the natural action of $f$ from $\mathbb{P}\left(V_{x}\right)$ to $\mathbb{P}\left(V_{f(x)}\right)$ to get a matrix whose elements enter into the definition of the Witten invariants. The problem then breaks up into two parts: (a) find the connection, and (b) prove that it is flat.

In Sect. 1 we consider the situation of a general symplectic manifold and give a cohomological identification of what such a connection should be. This is framed in holomorphic terms in what is really the Kodaira-Spencer deformation theory for a triple $(M, L, s)$ where $M$ is a complex manifold, $L$ a line bundle, and $s$ a section of $L$. A connection is given by a class in the first hypercohomology group of the complex of sheaves

$$
0 \rightarrow \mathscr{D}^{1}(L) \stackrel{s}{\longrightarrow} L \rightarrow 0
$$

where $\mathscr{D}^{1}(L)$ denotes the sheaf of first-order differential operators on $L$ and the map $s$ is evaluation on the section $s$. [This approach is inspired by a paper of Welters [20] who essentially deals with the abelian case $G=U(1)$.] The abstract cohomological formalism can be made quite explicit and elementary in Dolbeault terms, but it has the advantage of being accessible by other approaches - exact sequences and so forth - which enable us to find canonical classes in the required group.

In the second section we consider the specific case where the symplectic manifold $M$ is the space of equivalence classes of homomorphisms of the universal central extension $\Gamma$ of $\pi_{1}(\Sigma)$ into $S U(m)$. We consider this case initially since it is a smooth compact manifold. It corresponds for a choice of conformal structure on $\Sigma$ to the moduli space of stable bundles of rank $m$, degree 1 and fixed determinant. Some general properties of $M$ as a complex manifold are given including the identification of the holomorphic line bundle $L$. The principal result concerns the infinitesimal deformations of the Kähler polarizations we are concerned with. For a symplectic manifold in general, such an infinitesimal deformation is given by a complex symmetric tensor $G^{i j}$ satisfying an integrability condition. In our case this tensor is holomorphic and can be explicitly described. 
The connection is defined in Sect. 3. Formally speaking it arises from the coboundary map of a short exact sequence related to the sequence of sheaves

$$
0 \rightarrow \mathscr{D}^{1}(L) \rightarrow \mathscr{D}^{2}(L) \stackrel{\sigma}{\longrightarrow} S^{2} T \rightarrow 0,
$$

where $\mathscr{D}^{2}(L)$ is the sheaf of second order differential operators on $L, S^{2} T$ the sheaf of symmetric 2-tensors and $\sigma$ the symbol map. This formal description can be realized in two ways - either by a Dolbeault representative which uses the differential geometry of the Kähler manifold $M$, or by a Čech representative using a covering of $M$ by coordinate neighbourhoods. Either way, the connection may be described by a heat equation. Parallel translation with respect to the connection means evolving a section $s$ of $L$ along a curve by solving the heat equation. The differential geometric version is

$$
\frac{\partial s}{\partial t}=\frac{1}{(2 k+\lambda) \mathrm{i}} \sum_{i, j}\left(\nabla_{i}\left(G^{i j} \nabla_{j} s\right)+2 \mathrm{i} G^{i j} \frac{\partial F}{\partial z_{i}} \nabla_{j} s+\mathrm{i} k f_{G} s\right) .
$$

The holomorphic one is of the form

$$
\frac{\partial s}{\partial t}=\frac{1}{(2 k+\lambda) i} \sum_{i, j}\left(\frac{\partial}{\partial z_{i}}\left(G^{i j} \frac{\partial s}{\partial z_{j}}\right)+A_{G}^{i} \frac{\partial s}{\partial z_{i}}+B_{G} s\right),
$$

where the holomorphic coefficients apart from $G^{i j}$ depend on the choice of coordinates and trivialization of $L$.

In Sect. 4 we prove the flatness of the connection by using the holomorphic Čech description. The essential point is that the symmetric tensors $G^{i j}$, considered as functions on the cotangent bundle $T^{*} M$, actually Poisson-commute for general reasons. This becomes translated into commutation properties of the heat operators which define the connection (and whose symbols are the tensors $G^{i j}$ ) and hence of the covariant derivatives of the connection. The final section deals with the general case where the compact space $\operatorname{Hom}\left(\pi_{1}(\Sigma) ; S U(m)\right) / S U(m)$ is singular. Here, as in Sect. 4 we make some use of the moduli space of stable Higgs bundles a completion of the cotangent bundle $T^{*} M$ - to prove some basic holomorphic facts about $M$. Essentially, we work over the smooth open submanifold of irreducible connections and rely on Hartog's theorem to provide a substitute for compactness. One of the incidental outcomes of the holomorphic approach is to note that on the square root $K^{1 / 2}$ of the canonical bundle $K$ of $M$ there exists a natural space of globally defined commuting second-order differential operators. Its significance is, at least to the author, not clear - there is no connection to be defined for $K^{1 / 2}$ since it has no global sections.

There exist a number of other approaches to the flat connection, some arising from conformal field theory where this is a connection on the space of "conformal blocks." The description of Tsuchiya, Ueno, and Yamada [19] is also algebrogeometric in nature and, being phrased in the language of $\mathscr{D}$-modules shows how to extend the connection to stable curves. Allied to this is Segal's point of view [17] relating to representations of the loop group. The direct approach of Witten, Axelrod, and Della Pietra [22], viewing the problem as the relationship between the quantization of an affine space and its symplectic quotient, leads to the 
differential geometric heat equation above and shows that the connection introduced in a rather ad hoc manner in this paper is indeed the natural one.

Many aspects of the connection have so far resisted attack by the methods presented here, most notably the unitary structure which the vector space $V$ should have. Only in the abelian case does the heat operator preserve the inner product defined directly by the hermitian structure on $L$. Also, the behaviour of the space $V$ under degeneration of the conformal structure is not easily accessible. The method, or at least the basic ingredients, does however extend to the situation of surfaces with marked points - the starting point for Witten's knot invariants. The corresponding moduli space here is the space of parabolically stable vector bundles. It is easy to produce symmetric tensors and heat operators in this case, and indeed the explicit connection of Knizhnik and Zamolodzhikov $[9,8]$ can be viewed as a holomorphic heat equation. However, the modifications of the method given here to take into account the marked points are considerable and we have left such discussions until another occasion.

\section{Geometric Quantization}

The goal of the programme of geometric quantization is to associate to a symplectic manfiold a vector space, canonically defined up to a scalar factor, and satisfying certain properties. (The reader may refer to [23] or [5] for more details of this process.) The recognized method for producing this vector space requires the choice of a polarization of the symplectic manifold. The difficulty in practice in carrying out the quantization is in proving that, in an appropriate sense, the vector space is independent of that choice. Here, we shall consider this general problem for the case of a Kähler polarization.

Suppose $M^{2 n}$ is a compact symplectic manifold with symplectic form $\omega$. The symplectic form is a closed 2-form and thus defines a de Rham cohomology class

$$
[\omega] \in H^{2}(M ; \mathbb{R}) .
$$

If $\frac{1}{2 \pi}[\omega]$ is contained in the image of the integral cohomology

$$
H^{2}(M ; \mathbb{Z}) \rightarrow H^{2}(M ; \mathbb{R})
$$

then the symplectic form assumes a geometrical interpretation. It is the curvature form of a connection on a principal $U(1)$ bundle over $M$, whose first Chern class is $\frac{1}{2 \pi}[\omega]$. When $M$ is simply-connected, this connection is unique up to gauge equivalence.

Instead of considering the principal bundle, we may consider the associated $C^{\infty}$ complex line bundle $L$, which has a hermitian structure and a connection defined by a covariant derivative

$$
\nabla: \Omega^{0}(M ; L) \rightarrow \Omega^{1}(M ; L) .
$$


Now suppose we choose a Kähler polarization of the symplectic manifold $M$. This consists of an endomorphism $I \in \Omega^{0}(M ;$ End $T)$ of the tangent bundle of $M$ such that

$$
\begin{gathered}
I^{2}=-1, \\
{[I X, I Y]=[X, Y]+I[I X, Y]+I[X, I Y],} \\
\omega(X, I Y)=-\omega(I X, Y), \\
\omega(X, I X) \text { is positive definite. }
\end{gathered}
$$

By the well-known theorem of Newlander and Nirenberg the first two conditions give $M$ the structure of a complex manifold and the last two say that $g(X, Y)$ $=\omega(X, I Y)$ is a Kähler metric, with Kähler form $\omega \in \Omega^{1,1}(M)$.

With a Kähler polarization, we can give the $C^{\infty}$ line bundle $L$ the structure of a holomorphic line bundle by considering the differential operator

defined by

$$
\nabla^{0,1}: \Omega^{0}(M ; L) \rightarrow \Omega^{0,1}(M ; L)
$$

$$
\nabla^{0,1}=(1+i I) \nabla .
$$

[Recall that the complex forms of type $(0,1)$ are those on which $I$ acts as $-i$.]

In local terms, this is a differential operator of the form

$$
\sum_{i}\left(\frac{\partial f}{\partial \bar{z}_{i}}+\theta_{i} f\right) d \bar{z}_{i}
$$

and by the Dolbeault lemma, a local solution to

$$
\frac{1}{f} \frac{\partial f}{\partial \bar{z}_{i}}+\theta_{i}=0
$$

exists if and only if $\bar{\partial}\left(\Sigma \theta_{i} d \bar{z}_{i}\right)=0 \in \Omega^{0,2}(M ; \mathbb{R})$. This, however, is the $(0,2)$ component of the curvature of $\nabla$. In our case the curvature form is the Kähler form $\omega$ which is of type $(1,1)$. Thus the integrability condition is satisfied and the equation

$$
\nabla^{0,1} s=0
$$

has local non-vanishing solutions $s$. If $s$ and $\tilde{s}$ are two such solutions with $\tilde{s}=g s$, then from Eq. (1.6), $\frac{\partial g}{\partial \bar{z}_{i}}=0$, and so $g$ is a holomorphic transition function for the line bundle $L$. This gives it its holomorphic structure in the traditional sense. It means that a local holomorphic section $s$ is a solution of the equation $\nabla^{0,1} s=0$.

The space of global holomorphic sections of $L$ is by definition the vector space one takes as the quantization of $M$ relative to the Kähler polarization.

To each Kähler polarization I, we therefore associated the finite-dimensional vector space

$$
V_{I}=\left\{s \in \Omega^{0}(M ; L):(1+i I) \nabla s=0\right\} .
$$

We now need to address the question of the dependence of this vector space on the complex structure $I$. The first problem concerns the dimension of $V_{I}$ : is it constant as $I$ varies? 
To deal with this, recall that the Cauchy-Riemann operator $\nabla^{0,1}$ may be extended to act on $(0, p)$ forms with values in $L$ :

$$
\nabla^{0,1}: \Omega^{0, p}(M ; L) \rightarrow \Omega^{0, p+1}(M ; L)
$$

and the corresponding elliptic complex is the Dolbeault complex of the holomorphic line bundle $L$, with cohomology groups $H^{p}(M ; L)$. The vector space $V_{I}=H^{0}(M ; L)$ is the zeroth cohomology group of this complex. By Serre duality, (see e.g. [4])

$$
H^{p}(M ; L)^{*} \cong H^{n-p}\left(M ; K L^{-1}\right)
$$

where $K$ is the canonical bundle of holomorphic $n$-forms on $M$, and by the Kodaira-Nakano vanishing theorem [4] this space is zero for $p>0$ if the line bundle $K L^{-1}$ is negative, i.e. if the cohomology class $-c_{1}\left(K L^{-1}\right)=c_{1}(L)-c_{1}(K)$ is represented by a Kähler form. Since $c_{1}(L)$ is represented by the Kähler form $\frac{1}{2 \pi} \omega$ then for large enough $k,-c_{1}\left(K L^{-k}\right)=k c_{1}(L)-c_{1}(K)$ will be represented also by a Kähler form whatever $c_{1}(K)$ is. Thus replacing $L$ by $L^{k}$ (this change of level $k$ will be important later on) we will obtain vanishing in the general case. In particular cases, the relationship between $K$ and $L$ will yield more accurate information about the applicability of this argument.

This vanishing is relevant because the Riemann-Roch theorem [4] gives an expression for the alternating sum of dimensions

$$
\sum_{p=0}^{n}(-1)^{p} \operatorname{dim} H^{p}(M ; L)
$$

in terms of Chern classes which are deformation invariants of the polarization on $M$. Given the vanishing of $H^{p}(M ; L)$ for $p>0$ this yields the dimension of $V_{I}$, which must therefore be a deformation invariant too.

Suppose now that $N$ is a connected finite-dimensional parameter space of Kähler polarizations $I$. As $I$ varies over $N$, the constant-dimensional vector space $V_{I}$ defines, by elliptic regularity, a vector bundle $V$ over $N$. To say that the vector space $V_{I}$ is independent of $I$ is to say that given $I, J \in N$ there is a canonical identification of $V_{I}$ and $V_{J}$. Such an identification, if smoothly dependent on $I$ and $J$, can be interpreted as parallel translation of a connection on the vector bundle $V$ over $N$. Its independence of the path from $I$ to $J$ means it must be a flat connection. Conversely, a natural flat connection on $V$ will define, for $N$ simply-connected, an identification of $V_{I}$ and $V_{J}$ by parallel translation. This is the context, then, in which we seek to answer the question of dependence on polarization - to look for the infinitesimal version which manifests itself in the form of a flat connection. The fact that the vector space of a quantization should be defined only up to a scalar factor means that the projective spaces $\mathbb{P}\left(V_{I}\right)$ may be identified. This leads in general to a scalar ambiguity in the definition of the connection.

With this in mind, let us consider a path $I_{t}$ of Kähler polarizations and a smooth family $s_{t}$ of solutions to

$$
\nabla_{t}^{0,1} S_{t}=0
$$


Thus $s_{t}$ is a section of the vector bundle $V$ over the path. From (1.5) the differential equation takes the form

$$
\left(1+i I_{t}\right) \nabla s_{t}=0
$$

Differentiating, we obtain

$$
i \dot{I} \nabla s+(1+i I) \nabla \dot{s}=0 .
$$

A connection on $V$ will therefore be given by a $C^{\infty}$ section $u(s, \dot{I})$ of $L$, depending bilinearly on a holomorphic section $s$ and a tangent vector $\dot{I}$ to the space of Kähler polarizations, and satisfying the equation

$$
i{ } \nabla s+(1+i I) \nabla u=0 .
$$

Parallel translation along a path then consists of solving the equation

$$
\frac{\partial s}{\partial t}=u\left(s, \frac{\partial I}{\partial t}\right) .
$$

Note finally that since $\nabla^{0,1} s=0,(1.9)$ may be simplified to

$$
i i^{1,0} s+\nabla^{0,1} u=0 \text {. }
$$

We shall eventually give a cohomological interpretation of Eq. (1.10), but first let us consider what sort of tensorial object $\dot{I}$ is. It is an endomorphism of the tangent bundle but since $I^{2}=-1$, we have

$$
\dot{I}+I \dot{I}=0 .
$$

In other words $\dot{I}$ transforms the $-i$ eigenspace of $I$ to the $+i$ eigenspace, and hence

$$
\dot{I} \in \Omega^{0,1}(M ; T)
$$

where $T$ denotes the holomorphic vector bundle of $(1,0)$ tangent vectors. In local coordinates,

$$
\dot{I}=\sum_{i, j} a_{j}^{i} \frac{\partial}{\partial z_{i}} \otimes d \bar{z}_{j} .
$$

Linearizing the integrability condition (1.2) for $I$, we have

$$
\bar{\partial} \dot{I}=0 \in \Omega^{0,2}(M ; T) .
$$

Linearizing the compatibility condition with the symplectic form (1.3), we find that

$$
\dot{I}=\sum_{i, j, k} G^{i j} \omega_{j \bar{k}} \frac{\partial}{\partial z_{i}} \otimes d \bar{z}_{k},
$$

where $\omega=\sum_{j, k} \omega_{j \bar{k}} d z_{j} \wedge d \bar{z}_{k}$ is the Kähler form and

$$
G=\sum_{i, j} G^{i j} \frac{\partial}{\partial z_{i}} \otimes \frac{\partial}{\partial z_{j}}
$$

is a $C^{\infty}$ section of $T \otimes T$ which is symmetric (i.e. $G^{i j}=G^{i i}$ ). 
Denoting by $S^{2} T$ the bundle of symmetric tensors of type $(2,0)$, then we see that $i$ is defined by a section $G$ of $S^{2} T$, satisfying the integrability condition (1.12). In local terms this condition is:

$$
\sum_{j, k, l} \frac{\partial G^{i j}}{\partial \bar{z}_{l}} \omega_{j \bar{k}} d \bar{z}_{k} \wedge d \bar{z}_{l}=0
$$

since the symplectic form $\omega$ is closed.

Let us consider the question now of finding solutions $u(s, \dot{I})$ to Eq. (1.10). By the Dolbeault lemma a local solution will exist iff

$$
\bar{\partial}\left(i \nabla^{1,0} s\right)=0 \in \Omega^{0,2}(M ; L) .
$$

We check next that this condition does indeed hold. Using (1.13), the left-hand side is in local coordinates

$$
\sum_{i, j, k, l} \frac{\partial}{\partial \bar{z}_{l}}\left(\mathrm{i} G^{i j} \omega_{j \bar{k}} \nabla_{i} s \otimes d \bar{z}_{k} \wedge d \bar{z}_{l}\right)
$$

which, using (1.14) gives

$$
\sum_{i, j, k, l} \mathrm{i} G^{i j} \omega_{j \bar{k}} \frac{\partial}{\partial \bar{z}_{l}}\left(\nabla_{i} s\right) \otimes d \bar{z}_{k} \wedge d \bar{z}_{l} .
$$

But since the holomorphic structure on $L$ is defined by $\nabla^{0,1}$, then

$$
\frac{\partial}{\partial \bar{z}_{l}}\left(\nabla_{i} s\right)=\nabla_{\bar{l}} \nabla_{i} s=\nabla_{i} \nabla_{\bar{l}} s+\omega_{\overline{l i}} s,
$$

using the fact that the curvature of $\nabla$ on $L$ is $\omega$.

Since $s$ is also a holomorphic section of $L, \nabla_{l} S=0$, and so (1.15) may be written as

$$
-\sum \mathrm{i} G^{i j} \omega_{j \bar{k}} \omega_{i \bar{l}} s \otimes d \bar{z}_{k} \wedge d \bar{z}_{l} .
$$

But now the symmetry $G^{i j}=G^{j i}$ implies that this vanishes. Hence we can always find local solutions of (1.10).

This calculation can also be given a slightly different interpretation in terms of the first order differential operator $i I^{1,0}$. Written locally, we have

$$
\begin{aligned}
\mathrm{i} I \nabla^{1,0} s & =\sum_{i, j, k} \mathrm{i} G^{i j} \omega_{j \bar{k}} \nabla_{i} s \otimes d \bar{z}_{k} \\
& =\sum_{i, k} \alpha_{\bar{k}}^{\frac{i}{\partial}} \frac{\partial s}{\partial z_{i}} \otimes d \bar{z}_{k}+\sum_{k} \beta_{\bar{k}} s \otimes d \bar{z}_{k}
\end{aligned}
$$

in terms of a local holomorphic trivialization of $L$.

We now introduce the bundle $\mathscr{D}^{k}(L)$, defined as the vector bundle of holomorphic linear differential operators of order $k$ on $L$.

We can also identify it as the bundle $\operatorname{Hom}\left(J^{k}(L), L\right)$, where $J^{k}(L)$ is the vector bundle of $k$-jets of holomorphic sections of $L$. The bundles $\mathscr{D}^{k}(L)$ fit into short exact sequences of vector bundles:

$$
0 \rightarrow \mathscr{D}^{k-1}(L) \rightarrow \mathscr{D}^{k}(L) \stackrel{\sigma}{\longrightarrow} S^{k} T \rightarrow 0,
$$


where the homomorphism $\sigma$ is the principal symbol (or highest order term) of the operator.

With the above definition the local expression of $i I^{1,0}$ in (1.16) shows that it is an element of $\Omega^{0,1}\left(M ; \mathscr{D}^{1}(L)\right)$. The calculation above for the local integrability of Eq. (1.10) then shows that

$$
\bar{\partial}\left(i \dot{I}^{1,0}\right)=0 \in \Omega^{0,2}\left(M ; \mathscr{D}^{1}(L)\right) .
$$

To reinterpret (1.10) cohomologically we now introduce a complex which incorporates the basic data $i I^{1,0}$ and $u$ of the equation $-\mathrm{a}(0,1)$ form with values in $\mathscr{D}^{1}(L)$ and a $C^{\infty}$ section of $L$.

We set

$$
A^{p}=\Omega^{0, p}\left(M ; \mathscr{D}^{1}(L)\right) \oplus \Omega^{0, p-1}(M ; L),
$$

and define $d_{s}: A^{p} \rightarrow A^{p+1}$ by

$$
d_{s}(D, u)=\left(\bar{\partial} D, \bar{\partial} u+(-1)^{p-1} D s\right) .
$$

Since $\overline{\partial s}=0$, it is easily checked that $d_{s}^{2}=0$ and so we obtain a complex. The $p^{\text {th }}$ cohomology group of this complex we denote by

$$
\mathbb{H}_{s}^{p}\left(M ; \mathscr{D}^{1}(L)\right) .
$$

With this formalism we see that $\left(i I^{1,0}, u\right)$ can be viewed as an element of $A^{1}$ and the two equations (1.18) and (1.10) become

$$
d_{s}\left(i \nabla i \nabla^{1,0}, u\right)=0 .
$$

Thus any solution to (1.10) defines a cohomology class in $\mathbb{H}_{s}^{1}\left(M ; \mathscr{D}^{1}(L)\right)$. This cohomology group - a hypercohomology group of a double complex (see [20]) lies at the heart of the existence of the flat connection. It has the advantage of being viewed either from the Dolbeault point of view, as we have done here, or the Čech point of view which gives extra information as we shall see.

For the moment, note that the symbol map $\sigma$ [see (1.17)] applied to $D \in \Omega^{0,1}\left(M ; \mathscr{D}^{1}(L)\right)$ gives a homomorphism of cohomology groups

$$
\sigma: \mathbb{H}_{s}^{1}\left(M ; \mathscr{D}^{1}(L)\right) \rightarrow H^{1}(M ; T) .
$$

In our context, $\sigma\left(\dot{I} \nabla^{1,0}, u\right)=[\dot{I}]$ is the Kodaira-Spencer deformation class corresponding to the infinitesimal deformation $\dot{I}$ of the complex structure of $M$.

The Kähler form $\omega=\sum \omega_{i j} d z_{i} \wedge d \bar{z}_{j} \in \Omega^{0,1}\left(M ; T^{*}\right)$ also defines a cohomology class $[\omega] \in H^{1}\left(M ; T^{*}\right)$ and a cup product map

$$
[\omega]: H^{0}(M ; T) \rightarrow H^{1}(M ; \mathcal{O}),
$$

defined at the Dolbeault level by

$$
[\omega]\left(\sum_{i} X^{i} \frac{\partial}{\partial z_{i}}\right)=\sum_{i, j} X^{i} \omega_{i j} d \bar{z}_{j}
$$


for the holomorphic vector field $X=\sum_{i} X^{i} \frac{\partial}{\partial z_{i}} \in H^{0}(M ; T)$. Using this notation, the role of the hypercohomology group in defining a connection may be expressed by the following theorem:

Theorem (1.20). Let $M$ be a compact symplectic manifold and $L$ a line bundle over $M$ with connection whose curvature form is the symplectic form $\omega$. Suppose we have a family of Kähler polarizations of $M$ such that for each polarization

i) $[\omega]: H^{0}(M ; T) \rightarrow H^{1}(M ; \mathcal{O})$ is an isomorphism.

ii) For each holomorphic section $s \in H^{\circ}(M ; L)$ and tangent vector $\dot{I}$ to the family there exists a smoothly varying cohomology class

$$
A(\dot{I}, s) \in \mathbb{H}_{s}^{1}\left(M ; \mathscr{D}^{1}(L)\right)
$$

such that $-i \sigma A(\dot{I}, s)$ is the Kodaira-Spencer class $[\dot{I}] \in H^{1}(M ; T)$.

Then $A$ defines a connection on the bundle of projective spaces $\mathbb{P}\left(H^{0}(M ; L)\right)$ over the family. [As we shall see, condition (i) says that there are no holomorphic vector fields which fix the line bundle L.]

Proof. Firstly let us interpret condition (i) in the theorem. As in (1.17), the holomorphic bundle $\mathscr{D}^{1}(L)$ appears in an exact sequence

$$
0 \rightarrow \mathscr{D}^{0}(L) \rightarrow \mathscr{D}^{1}(L) \rightarrow T \rightarrow 0,
$$

where $\mathscr{D}^{0}(L) \cong \mathcal{O}$ is the trivial bundle of 0 -order differential operators. This exact sequence defines $\mathscr{D}^{1}(L)$ as an extension of $\mathcal{O}$ by $T$ and hence by a sheaf cohomology class in $H^{1}\left(M ; T^{*}\right)$. This is a multiple of the first Chern class

$$
\left[c_{1}(L)\right]=\frac{1}{2 \pi}[\omega] \in H^{1}\left(M ; T^{*}\right)
$$

by the Atiyah interpretation of characteristic classes [1].

Taking the exact cohomology sequence we have

$$
\begin{aligned}
0 & \rightarrow H^{0}(M ; \mathcal{O}) \rightarrow H^{0}\left(M ; \mathscr{D}^{1}(L)\right) \rightarrow H^{0}(M ; T) \stackrel{[\omega]}{\longrightarrow} H^{1}(M ; \mathcal{O}) \ldots \\
& \ldots \rightarrow H^{1}\left(M ; \mathscr{D}^{1}(L)\right) \rightarrow H^{1}(M ; T) \rightarrow H^{2}(M ; \mathcal{O}) \rightarrow \ldots
\end{aligned}
$$

Condition (i) tells us that $H^{0}(M ; \mathcal{O}) \cong H^{0}\left(M ; \mathscr{D}^{1}(L)\right)$, in other words that the only globally defined holomorphic first order operators on $L$ are multiplication operators by a holomorphic function. Since $M$ is compact these are just constants. It also says that the natural symbol map $H^{1}\left(M ; \mathscr{D}^{1}(L)\right) \rightarrow H^{1}(M ; T)$ is injective.

Suppose then we are given $A(\dot{I}, s) \in \mathbb{H}_{s}^{1}\left(M ; \mathscr{D}^{1}(L)\right)$ as in condition (ii) of the theorem. We represent the class by a 1 -cocycle

$$
(D, w) \in \Omega^{0,1}\left(M ; \mathscr{D}^{1}(L)\right) \oplus \Omega^{0}(M ; L) .
$$

Since $-i \sigma(D) \in \Omega^{0,1}(M ; T)$ is cohomologous to $\dot{I}$ then $D$ and $i I^{1,0}$ have cohomologous symbols and hence by the above injectivity are cohomologous in $\Omega^{0,1}\left(M ; \mathscr{D}^{1}(L)\right)$, i.e. there exists $P \in \Omega^{0}\left(M ; \mathscr{D}^{1}(L)\right)$ such that

$$
D-i \bar{I}^{1,0}=\bar{\partial} P .
$$


Since $d_{s}(D, w)=0$,

$$
D s+\bar{\partial} w=i \bar{I}^{1,0} s+\bar{\partial}(P s+w)=0,
$$

and hence $u=P s+w$ gives a solution to (1.10).

Let $u_{1}, u_{2}$ be solutions to $(1.10)$ such that $\left(i I^{1,0}, u_{1}\right)$ and $\left(i \dot{I}^{1,0}, u_{2}\right)$ are cohomologous in $\mathbb{H}_{s}^{1}\left(M ; \mathscr{D}^{1}(L)\right)$. Then

$$
\left(0, u_{1}-u_{2}\right)=d_{s} P, \quad P \in \Omega^{0}\left(M ; \mathscr{D}^{1}(L)\right) .
$$

From the definition of $d_{s}$ this means that

$$
\bar{\partial} P=0
$$

and

$$
u_{2}-u_{1}=P s \text {. }
$$

However condition (i) says that the only global holomorphic sections of $\mathscr{D}^{1}(L)$ are constants, so $P$ is a constant $c$ and $u_{2}=u_{1}+c s$, i.e. $u$ is well-defined up to a multiple of $s$. This ambiguity is precisely the indeterminacy to obtain a connection on the projective bundle rather than the vector bundle.

Note that this uniqueness also gives the bilinearity of the dependence of $u$ on $\dot{I}$ and $s$.

This is as far as the general theory of Kähler polarizations will take us. We consider next the more specific symplectic manifolds that arise from representations of surface groups.

\section{Spaces of Representations}

The manifolds to which we shall apply the above process of geometric quantization are spaces of equivalence classes of representations of the fundamental group of a compact oriented surface $\Sigma$ of genus $g>1$ into a compact Lie group $G$. We consider then the space

$$
\operatorname{Hom}\left(\pi_{1}(\Sigma) ; G\right)
$$

of homomorphisms from the fundamental group $\pi_{1}(\Sigma)$ to $G$ and the quotient space

$$
\operatorname{Hom}\left(\pi_{1}(\Sigma) ; G\right) / G
$$

by the conjugation action of $G$.

Since $\pi_{1}(\Sigma)$ is a group with $2 g$ generators $A_{1}, B_{1}, \ldots, A_{g}, B_{g}$ satisfying the one relation $\prod_{i=1}^{g}\left[A_{i}, B_{i}\right]=1$, this compact space has an explicit description, but unfortunately is rarely a manifold because of the existence of reducible representations and in particular the trivial one. At an irreducible representation it is smooth, with tangent space the cohomology group $H^{1}\left(\pi_{1}(\Sigma) ; g\right)$ - the cohomology of the Lie algebra of $G$ considered as a $\pi_{1}(\Sigma)$-module.

There are, however, compact spaces of representations which are smooth manifolds (see [2]) if we replace the fundamental group $\pi_{1}(\Sigma)$ by its universal central extension

$$
0 \rightarrow \mathbb{Z} \rightarrow \Gamma \rightarrow \pi_{1}(\Sigma) \rightarrow 1
$$


generated by $A_{1}, B_{1}, \ldots, A_{g}, B_{g}$ and the central element $J$ satisfying $\prod_{i=1}^{g}\left[A_{i}, B_{i}\right]=J$. The space of equivalence classes of representations of $\Gamma$ into $S U(m)$ such that $J$ maps to a generator of the centre $\mathbb{Z} / m$ is a smooth compact manifold $M$.

The spaces we have just defined as manifolds are not yet canonically symplectic manifolds. This additional fact becomes apparent by adopting the point of view of Atiyah and Bott [2] and interpreting via the holonomy representation the above spaces as spaces of gauge-equivalence classes of flat connections over $\Sigma$. If $P$ is a principal $G$-bundle over $\Sigma$, then the infinite-dimensional affine space $\mathscr{A}$ of all connections on $P$ is canonically a symplectic manifold: a tangent vector to $\mathscr{A}$ is a Lie-algebra-valued 1-form $\alpha \in \Omega^{1}(\Sigma ; \operatorname{ad} P)$ and

$$
\omega\left(\alpha_{1}, \alpha_{2}\right)=\int_{\Sigma} B\left(\alpha_{1} \wedge \alpha_{2}\right)
$$

defines a non-degenerate skew form where $B$ is a bi-invariant positive definite inner product on the Lie algebra. For $G=S U(m)$, we have

$$
\omega\left(\alpha_{1}, \alpha_{2}\right)=-\int_{\Sigma} \operatorname{Tr}\left(\alpha_{1} \wedge \alpha_{2}\right) .
$$

Since $\omega$ is a constant form it is closed. The infinite-dimensional group $\mathscr{G}$ of gauge transformations (automorphisms of $P$ ) acts on $\mathscr{A}$ preserving the symplectic form $\omega$ and has moment map

$$
\mu: \mathscr{A} \rightarrow \operatorname{Lie}(\mathscr{G})^{*} \cong \Omega^{0}(\Sigma ; \operatorname{ad} P)^{*},
$$

defined by

$$
\mu(A)=F_{A} \in \Omega^{2}(\Sigma ; \operatorname{ad} P) .
$$

The reduced phase space, or Marsden-Weinstein quotient

$$
\mu^{-1}(0) / \mathscr{G}
$$

then has an induced symplectic structure. This space is precisely the set of gaugeequivalence classes of connections $A$ with $F_{A}=0$, i.e. flat connections, and hence a space of representations of $\pi_{1}(\Sigma)$. The central extension case considered above may similarly be interpreted either as the gauge equivalence classes of $U(m)$ connections with central curvature or via a refined notation of equivalence for flat $P U(m)-(=U(m) /$ centre $)$-connections. In either case the symplectic formalism goes through and we are left with a symplectic manifold $(M, \omega)$ of equivalence classes of representations of an abstract group.

With the flat connection interpretation, the tangent space to $M$ is the first cohomology group $H_{A}^{1}(\Sigma ; \operatorname{ad} P)$ of the complex

$$
\Omega^{0}(\Sigma ; \operatorname{ad} P) \stackrel{d_{A}}{\longrightarrow} \Omega^{1}(\Sigma ; \operatorname{ad} P) \stackrel{d_{A}}{\longrightarrow} \Omega^{2}(\Sigma ; \operatorname{ad} P)
$$

defined by the covariant exterior derivative $d_{A}$. The symplectic form is obtained by applying (2.1) to any two representatives $\alpha_{1}, \alpha_{2}$ of $H^{1}(\Sigma ; \operatorname{ad} P)$. Since

$$
\omega\left(d_{A} \psi, \alpha\right)=\int_{\Sigma} B\left(d_{A} \psi \wedge \alpha\right)=\int_{\Sigma} d B(\psi, \alpha)=0,
$$

the result is independent of the choice of representative. 
By irreducibility of the connection the zeroth cohomology group of (2.3) vanishes and then by duality and the index theorem,

$$
\begin{aligned}
\operatorname{dim} M & =\operatorname{dim} H_{A}^{1}(\Sigma ; \operatorname{ad} P) \\
& =2 g \quad \text { for } \quad G=U(1) \\
& =(2 g-2) \operatorname{dim} G \text { for } G \text { semi-simple. }
\end{aligned}
$$

There is a natural way to produce a Kähler polarization of this symplectic manifold and that is to choose a conformal structure on the surface $\Sigma$ and make it into a Riemann surface. This consists of the choice of a Hodge star-operator * (or complex structure $I$ ) on $\Omega^{1}(\Sigma)$ :

$$
\begin{gathered}
*: \Omega^{1}(\Sigma) \rightarrow \Omega^{1}(\Sigma), \\
*^{2}=-1 .
\end{gathered}
$$

With this choice each cohomology class in $H_{A}^{1}(\Sigma ; \operatorname{ad} P)$ has by Hodge theory a unique harmonic representative $\alpha$ :

$$
\left\{\begin{array}{l}
d_{A} \alpha=0, \\
d_{A} * \alpha=0 .
\end{array}\right.
$$

We define an endomorphism $I$ on this space of harmonic forms by

$$
I \alpha=-* \alpha \text {. }
$$

Since for any 1-form $\beta \in \Omega^{1}(\Sigma)$,

$$
(* \beta) \wedge \beta=-\beta \wedge * \beta \geqq 0,
$$

then

$$
-\omega(I \alpha, \alpha)=\omega(\alpha, I \alpha)=-\int_{\Sigma} B(\alpha \wedge * \alpha) \geqq 0
$$

and conditions (1.1),(1.3), and (1.4) for a Kähler polarization are then easily seen to hold.

This particular choice of $I$ defines by the same argument a Kähler polarization on the infinite-dimensional symplectic manifold $\mathscr{A}$, making it into a complex affine space. For general reasons this induces an integrable complex structure on the quotient, satisfying the condition (1.2) for a polarization of $M$.

In fact, there is a much more explicit description of this complex structure. For the moment, to place ourselves firmly in the context of smooth manifolds, let us assume that $M$ is the compact manifold of equivalence classes of representations of $\Gamma$ into $S U(m)$ with $J$ generating the centre of $S U(m)$, denoted by $\operatorname{Hom}_{1}(\Gamma, S U(m)) / S U(m)$. Then $M$ is the space of gauge-equivalence classes of connections on a fixed rank $m$ hermitian vector bundle $E$ of degree one, and with fixed central curvature.

The complex structure $I$ on $\mathscr{A}$ identifies $\mathscr{A}$ as the complex affine space of Cauchy-Riemann operators on $E$ obtained by taking the $(0,1)$ part of the connection just as in (1.5) - the space $\Omega^{0,1}(\Sigma ; \operatorname{ad} P)$ is by the definition of $I$ the space of tangent vectors to $\mathscr{A}$ of type $(1,0)$. 
Restricting to the connections with central curvature, the theorem of Narasimhan and Seshadri (see $[2,12]$ ) provides the explicit description of $M$ as a complex manifold. This theorem states that a holomorphic vector bundle $E$ on a compact Riemann surface $\Sigma$ is stable if and only if there exists a unitary connection with central curvature compatible with the $\bar{\partial}$-operator on $E$. Here stability means that for each holomorphic subbundle $U \subset E$,

$$
\frac{\operatorname{deg} U}{\operatorname{rank} U}<\frac{\operatorname{deg} E}{\operatorname{rank} E}
$$

It follows, as discussed in [2], that the moduli space of equivalence classes of stable bundles of rank $m$, degree one and fixed determinant can be naturally identified with the holomorphic structure on $M$ defined by the Kähler polarization above. It is a smooth projective variety of complex dimension $n=(g-1)\left(m^{2}-1\right)$ [cf. (2.4)].

Of course, for $G=U(1)$, we obtain also a smooth manifold. With this polarization it is just a component of the group of holomorphic line bundles on $\Sigma$ the Picard variety. Each component is isomorphic to the complex torus $H^{1}(\Sigma ; \mathcal{O}) / H^{1}(\Sigma ; \mathbb{Z})$.

We need now to establish some basic properties of $M^{n}$ as a Kähler manifold. The first is to identify the line bundle $L$ of Sect. 1, and its holomorphic structure relative to the polarization. Quillen's work [15] on determinants of CauchyRiemann operators does this - the Kähler form on $\mathscr{A}$ defined above is the curvature of a unitary connection on the determinant bundle of the universal family of Cauchy-Riemann operators

$$
\bar{\partial}_{A}: \Omega^{0}(\Sigma ; E) \rightarrow \Omega^{0,1}(\Sigma ; E)
$$

parametrized by $\mathscr{A}$.

Replacing $E$ by ad $P=\operatorname{End}_{0} E$, the bundle of trace zero endomorphisms of $E$, we obtain another determinant line bundle for the family of operators

$$
\bar{\partial}_{A}: \Omega^{0}\left(\Sigma ; \operatorname{End}_{0} E\right) \rightarrow \Omega^{0,1}\left(\Sigma ; \operatorname{End}_{0} E\right)
$$

whose curvature is a positive integer multiple $\lambda \omega$ of the symplectic form. The integer $\lambda$ may be calculated (see [2]) by the Grothendieck-Riemann-Roch theorem or its formal equivalent.

These two properties pass to the symplectic quotient $M$, so we may identify the line bundle $L$ with the determinant bundle for the family of stable $\bar{\partial}_{A}$-operators. In the stable situation there are no holomorphic sections of $\operatorname{End}_{0} E$ (a vanishing theorem using the flat connection will prove this) so the determinant bundle for (2.7) is the line bundle $\Lambda^{n} H^{1}\left(\Sigma ; \operatorname{End}_{0} E\right)$. However $H^{1}\left(\Sigma ; \operatorname{End}_{0} E\right)$ is represented by the harmonic solutions to $(2.5)$ of type $(0,1)$, and thus using the complex structure $I$ is the holomorphic tangent space. Thus the canonical bundle $K$ of holomorphic $n$-forms on $M^{n}$ is related to the line bundle $L$ by

$$
K \cong L^{-\lambda} \text {. }
$$

From this we see that for every $k>0$, the line bundle $K L^{-k}=L^{-(\lambda+k)}$ is negative and so in particular the Kodaira-Nakano vanishing theorem guarantees that 
$H^{0}\left(M ; L^{k}\right)$ has constant dimension for Kähler polarizations of this type. Taking $k=0$ it also tells us that

$$
H^{1}(M ; \mathcal{O})=0 .
$$

There are two further properties of $M$ we shall need, which were established by Narasimhan and Ramanan [11]. The first is the non-existence of holomorphic vector fields

$$
H^{0}(M ; T)=0 .
$$

The second concerns the infinitesimal deformation map $\varrho: H^{1}\left(\Sigma ; K^{-1}\right)$ $\rightarrow H^{1}(M ; T)$ which associates to each deformation of complex structure on $\Sigma$ the corresponding deformation of complex structure of $M$ :

$$
\varrho: H^{1}\left(\Sigma ; K^{-1}\right) \rightarrow H^{1}(M ; T) \text { is injective . }
$$

We shall in Sect. 5 give independent proofs of these results when we deal with the case where $M$ is singular.

Having reached the point of considering deformations of complex structure, we should address now the question of finding the symmetric tensor $G$ which gives rise as in (1.13) to the infinitesimal deformation of a Kähler polarization of $M$.

An infinitesimal deformation of the conformal structure * on $\Sigma$ is [cf. (1.11)] a Beltrami differential

$$
\dot{*}=a \frac{d}{d z} \otimes d \bar{z} \in \Omega^{0,1}\left(\Sigma ; K^{-1}\right) .
$$

We have from (2.5) and (2.6) that the $(1,0)$ tangent vectors at a point of $M$ are the $d_{A}$-harmonic forms in $\Omega^{0,1}\left(\Sigma ; \operatorname{End}_{0} E\right)$ and thus representatives for classes in the Dolbeault cohomology $H^{1}\left(\Sigma ; \operatorname{End}_{0} E\right)$. Similarly the $(0,1)$ vectors are harmonic forms in $\Omega^{1,0}\left(\Sigma ; \operatorname{End}_{0} E\right)$, i.e. holomorphic sections of $\operatorname{End}_{0} E \otimes K$ over $\Sigma$. Thus

$$
\begin{gathered}
T^{1,0} \cong H^{1}\left(\Sigma ; \operatorname{End}_{0} E\right), \\
T^{0,1} \cong H^{0}\left(\Sigma ; \operatorname{End}_{0} E \otimes K\right),
\end{gathered}
$$

and as in (1.11), if $\dot{I}$ is the infinitesimal deformation of complex structure of $M$,

$$
\dot{I}: T^{0,1} \rightarrow T^{1,0} .
$$

Lemma (2.13). If $X \in T^{0,1}$ is given by a holomorphic section $\alpha$ of $\operatorname{End}_{0} E \otimes K$, then $\dot{I} X \in T^{1,0}$ is represented in $H^{1}\left(\Sigma ; \operatorname{End}_{0} E\right)$ by

$$
-* * \alpha \in \Omega^{0,1}\left(\Sigma ; \operatorname{End}_{0} E\right) \text {. }
$$

Proof. Consider a 1-parameter family of deformations $*(t)$ of conformal structure on $\Sigma$ and the corresponding family $I(t)$ of complex structures on $M$. Then $\alpha \in \Omega^{1}\left(\Sigma ; \operatorname{End}_{0} E\right)$ can be written as

$$
\alpha=h+d_{A} \psi
$$

for some form $h$ harmonic with respect to ${ }^{*}$. Differentiating at $t=0$,

$$
0=\dot{h}+d_{A} \dot{\psi} \text { and } h=\alpha \text {. }
$$


Now by the definition of $I$,

$$
I[\alpha]=[-* h] \in H_{A}^{1}\left(\Sigma ; \operatorname{End}_{0} E\right) .
$$

Hence differentiating

$$
\begin{aligned}
\dot{I}[\alpha] & =[-\dot{*} h-* \dot{h}] \\
& =\left[-\dot{*} \alpha+* d_{A} \dot{\psi}\right] \quad \text { from (2.14). }
\end{aligned}
$$

But now $\dot{I}$ transforms $T^{0,1}$ to $T^{1,0}$, thus this class is represented by a harmonic $(0,1)$-form $\beta$, i.e. there exists $\phi \in \Omega^{0}\left(\Sigma ; \operatorname{End}_{0} E\right)$ such that

$$
-* \alpha+* d_{A} \dot{\psi}+d_{A} \phi=\beta \in \Omega^{0,1}\left(\Sigma ; \operatorname{End}_{0} E\right) \text {. }
$$

Since $* \in \Omega^{0,1}\left(\Sigma ; K^{-1}\right)$ and $\alpha \in \Omega^{0}\left(\Sigma ; \operatorname{End}_{0} K\right), \dot{*} \alpha$ is already of type $(0,1)$, so

$$
\beta=-\dot{*} \alpha+\bar{\partial}_{A}(\phi-i \dot{\psi})
$$

and

$$
[\beta]=[-\dot{*} \alpha] \in H^{1}\left(\Sigma ; \operatorname{End}_{0} E\right)
$$

which proves the lemma.

To find the symmetric tensor $G$ corresponding to $\dot{I}$ all we need to do from (1.13) is to use the symplectic form to give an isomorphism

$$
\left(T^{1,0}\right) * \stackrel{\omega^{-1}}{\longrightarrow} T^{0,1}
$$

and then compose with $\dot{I}: T^{0,1} \rightarrow T^{1,0}$. The isomorphism

$$
\omega^{-1}: H^{1}\left(\Sigma ; \operatorname{End}_{0} E\right)^{*} \rightarrow H^{0}\left(\Sigma ; \operatorname{End}_{0} E \otimes K\right)
$$

is however precisely Serre duality - the bilinear pairing

$$
H^{0}\left(\Sigma ; \operatorname{End}_{0} E \otimes K\right) \otimes H^{1}\left(\Sigma ; \operatorname{End}_{0} E\right) \rightarrow H^{1}(\Sigma ; K) \cong \mathbb{C}
$$

defined by

$$
\int_{\Sigma} \operatorname{Tr}(\alpha \wedge \beta)
$$

Thus, considering $G$ as a quadratic function on the holomorphic cotangent bundle

$$
T^{*}=\left(T^{1,0}\right)^{*} \cong H^{0}\left(\Sigma ; \operatorname{End}_{0} E \otimes K\right)
$$

we have from Lemma (2.13)

$$
G(\alpha, \alpha)=\int_{\Sigma} \operatorname{Tr}(\alpha \wedge * \alpha)=\int_{\Sigma}\left(\operatorname{Tr} \alpha^{2}\right) \dot{*}
$$

where $\operatorname{Tr} \alpha^{2} \in H^{0}\left(\Sigma ; K^{2}\right)$ is, for $\alpha \in H^{0}\left(\Sigma ;\right.$ End $\left._{0} E \otimes K\right)$ a holomorphic quadratic differential.

Note that $G$ only depends on the cohomology class of $* \in \Omega^{0,1}\left(\Sigma ; K^{-1}\right)$ in $H^{1}\left(\Sigma ; K^{-1}\right)$ - the Kodaira-Spencer class-and depends holomorphically on $M$ from its cohomological description. It is clear also, ignoring the origins of $G$, that any holomorphic symmetric tensor will satisfy the integrability condition (1.14) and so give an infinitesimal deformation of Kähler polarization. 
We put this result more formally as follows:

Proposition (2.16). The cup product map

$$
H^{1}\left(\Sigma ; K^{-1}\right) \otimes H^{0}\left(\Sigma ; \operatorname{End}_{0} E \otimes K\right) \rightarrow H^{1}\left(\Sigma ; \operatorname{End}_{0} E\right)
$$

defines for each infinitesimal deformation of conformal structure of $\Sigma$ a holomorphic symmetric tensor $G$ on the moduli space of stable bundles $M$ which is the corresponding infinitesimal deformation of the Kähler polarization.

\section{The Connection}

In the previous section we saw that the infinitesimal deformation coming from a standard Kähler polarization on the symplectic manifold

$$
M=\operatorname{Hom}_{1}(\Gamma ; S U(m)) / S U(m)
$$

arose from a holomorphic symmetric tensor

$$
G \in H^{0}\left(M ; S^{2} T\right) \text {. }
$$

On the other hand Theorem (1.20) says that to define a connection on the vector bundle $V$ over a family of Kähler polarizations we need a class in the hypercohomology group $\mathbb{H}_{s}^{1}\left(M ; \mathscr{D}^{1}(L)\right)$.

There is a canonical way of associating to a holomorphic symmetric tensor such a class. It stems from the following short exact sequence of (very short!) complexes of sheaves

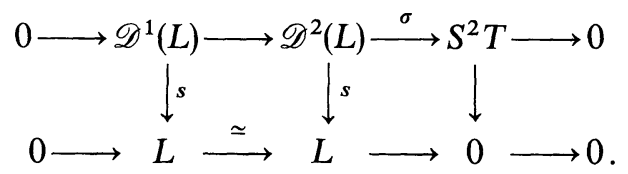

The top row is just the sequence (1.17) for the sheaf $\mathscr{D}^{2}(L)$ of second order operators on $L$ and the vertical homomorphisms consist of evaluating the differential operator on a given section $s$ of $L$. There is a corresponding long exact sequence of hypercohomology groups

$$
0 \rightarrow \mathbb{H}_{s}^{0}\left(M ; \mathscr{D}^{1}(L)\right) \rightarrow \mathbb{H}_{s}^{0}\left(M ; \mathscr{D}^{2}(L)\right) \stackrel{\sigma}{\longrightarrow} H^{0}\left(M ; S^{2} T\right) \stackrel{\delta}{\longrightarrow} \mathbb{H}_{s}^{1}\left(M ; \mathscr{D}^{1}(L)\right) \rightarrow
$$

It is the coboundary map $\delta$ which concerns us here. There are two ways to go from this abstract cohomology formalism to explicit formulas - we can represent cohomology classes by either Dolbeault or Čech representatives. Let us first consider the Dolbeault version of (3.2). We also introduce the level $k$ - that is, we try to quantize the symplectic manifold $M$ with symplectic form $k \omega$ for a positive integer $k$. This means replacing the line bundle $L$ by $L^{k}$.

Now from the definition of the complex (1.19) which defines the hypercohomology groups $\mathbb{H}_{s}^{p}$,

$$
A^{0}=\Omega^{0}\left(M ; \mathscr{D}^{i}(L)\right)
$$


Thus to describe $\delta(G)$, for $G \in H^{0}\left(M ; S^{2} T\right)$ in Dolbeault terms, we choose a $C^{\infty}$ section $\Delta$ of $\mathscr{D}^{2}(L)$ such that $\sigma(\Delta)=G$, and then consider

$$
d_{s}(\Delta, 0)=(\bar{\partial} \Delta,-\Delta s) .
$$

Since the principal symbol of the second order operator $\Delta$ is holomorphic, $\bar{\partial} \Delta$ is first order, an element of $\Omega^{0,1}\left(M ; \mathscr{D}^{1}(L)\right)$ and (3.3) defines the class

$$
\delta(G) \in \mathbb{H}_{s}^{1}\left(M ; \mathscr{D}^{1}(L)\right) .
$$

Using the connection $\nabla$ on $L^{k}$ and the Levi-Civita connection on the Kähler manifold $M$, there is a natural choice for $\Delta$ - the complex Laplace-Beltrami operator

$$
\Delta s=\sum_{i, j} \nabla_{i}\left(G^{i j} \nabla_{j} s\right) .
$$

This is a second-order differential operator on $L^{k}$ with symbol $G$ as required.

To find the hypercohomology class we take $\bar{\partial} \Delta$ or equivalently we use the $(0,1)$ part of the connection on the tangent bundle $T$ of $M$ and $L^{k}$. From (3.4) we have for any local holomorphic section $s$,

$$
\nabla_{i}(\Delta s)=\sum_{i, j} \nabla_{i} \nabla_{i}\left(G^{i j} \nabla_{j} s\right) .
$$

Now $\sum G^{i j} \nabla_{j} s \otimes \frac{\partial}{\partial z_{i}}$ is a section of $L^{k} \otimes T$. The curvature of the connection on this bundle gives

$$
\sum_{i, j} \nabla_{\bar{l}} \nabla_{i}\left(G^{i j} \nabla_{j} s\right)=\sum_{i, j}\left(R_{\overline{l i}}+k \omega_{\overline{l i}}\right) G^{i j} \nabla_{j} s+\sum_{i, j} \nabla_{i} \nabla_{\bar{l}}\left(G^{i j} \nabla_{j} s\right)
$$

where $R_{i \bar{j}}$ is the Ricci form.

But

$$
\begin{aligned}
\sum_{i, j} \nabla_{i} \nabla_{l}\left(G^{i j} \nabla_{j} s\right) & =\sum_{i, j} \nabla_{i}\left(G^{i j} \nabla_{i} \nabla_{j} s\right), \quad \text { since } G \text { is holomorphic } \\
& =\sum_{i, j} \nabla_{i}\left(G^{i j} k \omega_{i j} s\right),
\end{aligned}
$$

since $s$ is holomorphic and the curvature of $\nabla$ on $L^{k}$ is $k \omega$. Hence, finally

$$
\nabla_{\bar{l}}(\Delta s)=\sum_{i, j}\left(R_{\overline{l i}}+2 k \omega_{\overline{l i}}\right) G^{i j} \nabla_{j} s+k \sum_{i, j}\left(\nabla_{i} G^{i j}\right) \omega_{\bar{l} j} s .
$$

We can now prove the following:

Theorem (3.6). The class $\frac{\delta(G)}{(2 k+\lambda) i} \in \mathbb{H}_{s}^{1}\left(M ; \mathscr{D}^{1}\left(L^{k}\right)\right)$ defines via Theorem (1.20) a connection on the bundle of projective spaces $\mathbb{P}\left(H^{0}\left(M ; L^{k}\right)\right)$ over the family of Kähler polarizations arising from a family of conformal structures on $\Sigma$.

Proof. First note that from (2.9) and (2.10), condition (i) of Theorem (1.20) holds. It also holds in the abelian situation $M=H^{1}(\Sigma ; \mathcal{O}) / H^{1}(\Sigma ; \mathbb{Z})$, where the symplectic form $\omega$ is constant and positive definite. 
If we take $\frac{\delta(G)}{(2 k+\lambda) i}=A(\dot{I}, s)$ in the theorem, then to obtain a connection we simply have to check that

$$
\frac{-\sigma \delta(G)}{(2 k+\lambda)} \in H^{1}(M ; T)
$$

is the Kodaira-Spencer class.

From (2.13) and (2.15) the Kodaira-Spencer class is represented by

$$
\dot{I}=\sum_{i, j, k} G^{i j} \omega_{j \bar{k}} \frac{\partial}{\partial z_{i}} \otimes d \bar{z}_{k},
$$

whereas from $(3.5), \sigma \delta(G)$ is represented by

$$
-\sum_{i, j, k} G^{i j}\left(2 k \omega_{j \bar{k}}+R_{j \bar{k}}\right) \frac{\partial}{\partial z_{i}} \otimes d \bar{z}_{k} .
$$

On the other hand, the Ricci form

$$
\frac{1}{2 \pi} \sum_{j, k} R_{j \bar{k}} d z_{j} \wedge d \bar{z}_{k}
$$

represents the first Chern class of $M$, and from (2.8) this is cohomologous to $\lambda \omega$. Thus $\sigma \delta(G)$ is also represented by

i.e.

$$
-(2 k+\lambda) \sum_{i, j, k} G^{i j} \omega_{j \bar{k}} \frac{\partial}{\partial z_{i}} d \bar{z}_{k},
$$

$$
-\sigma \delta(G)=(2 k+\lambda)[\dot{I}] \in H^{1}(M ; T),
$$

thus proving the theorem.

We can, in fact, be a little more explicit about the form of this connection. Firstly, since the Ricci form and $\lambda \omega$ are cohomologous we may define a real function - the Ricci potential $F$ characterized by

$$
R_{j \bar{k}}-\lambda \omega_{j \bar{k}}=2 i \frac{\partial^{2} F}{\partial z_{j} \partial \bar{z}_{k}}
$$

and normalized by the condition that its integral over $M$ is zero. Thus (3.5) can be written as

$$
\nabla_{\bar{l}}(\Delta s)=(2 k+\lambda) \sum_{i, j} G^{i j} \omega_{j \bar{l}} \nabla_{i} s-2 \mathrm{i} \sum_{i, j} G^{i j} \frac{\partial^{2} F}{\partial z_{i} \partial \bar{z}_{l}} \nabla_{j} s-k \sum_{i, j}\left(\nabla_{i} G^{i j}\right) \omega_{j l} s,
$$

and so, since $G$ is holomorphic,

$$
\begin{aligned}
& \nabla_{\bar{l}}\left(\Delta s+2 \mathrm{i} \sum G^{i j} \frac{\partial F}{\partial z_{i}} \nabla_{j} s\right) \\
& =(2 k+\lambda) \sum_{i, j} G^{i j} \omega_{j i} \nabla_{i} s+2 \mathrm{i} \sum_{i, j} G^{i j} \frac{\partial F}{\partial z_{i}} \nabla_{l} \nabla_{j} s-k \sum_{i, j}\left(\nabla_{i} G^{i j}\right) \omega_{j l} s \\
& =-(2 k+\lambda) \sum_{i, j} G^{i j} \omega_{j i} \nabla_{i} s-2 \mathrm{i} k \sum_{i, j} G^{i j} \omega_{j \bar{l}} \frac{\partial F}{\partial z_{i}} s-k \sum_{i, j}\left(\nabla_{i} G^{i j}\right) \omega_{j i} s
\end{aligned}
$$


The $(0,1)$ form

$$
\theta_{G}=\sum_{i, j}\left(2 i G^{i j} \omega_{j l} \frac{\partial F}{\partial z_{i}}+\left(\nabla_{i} G^{i j}\right) \omega_{j i}\right)
$$

is $\bar{\partial}$-closed from its origins in Theorem (1.20) and since $H^{1}(M ; \mathcal{O})=0$, there is a function $f_{G}$, uniquely defined up to an additive constant, such that

$$
\bar{\partial} f_{G}=\theta_{G} \text {. }
$$

Thus (3.10) may be written as

$$
\nabla_{i}\left(\Delta s+2 \mathrm{i} \sum_{i, j} G^{i j} \frac{\partial F}{\partial z_{i}} \nabla_{j} s+k f_{G} s\right)=-(2 k+\lambda) \sum_{i, j} G^{i j} \omega_{j i} \nabla_{i} s,
$$

i.e.

where

$$
\mathrm{i} I \nabla^{1,0} s=\mathrm{i} \sum_{i, j} G^{i j} \omega_{j i} \nabla_{i} s=\nabla^{0,1} u
$$

$$
u(\dot{I}, s)=-\frac{1}{(2 k+\lambda)}\left\{\mathrm{i} \sum_{i, j}\left(\nabla_{i}\left(G^{i j} \nabla_{j} s\right)-2 G^{i j} \frac{\partial F}{\partial z_{i}} \nabla_{j} s+\mathrm{i} k f_{G} s\right)\right\} .
$$

From (1.9), parallel translation with respect to this connection then consists of solving the differential-geometric heat equation

$$
\frac{\partial s}{\partial t}=-\frac{1}{(2 k+\lambda)} \sum_{i, j}\left(i \nabla_{i}\left(G^{i j} \nabla_{j} s\right)-2 G^{i j} \frac{\partial F}{\partial z_{i}} \nabla_{j} s+i k f_{G} s\right) .
$$

(3.14) Remarks. 1. In the abelian case, then a simplification occurs. First of all, the natural Kähler metric on the Jacobian torus is flat, so the Ricci tensor is zero and $\lambda=0$. Secondly, the holomorphic sections of $S^{2} T$ are just quadratic expressions in global holomorphic vector fields and as such are covariant constant. Consequently (3.5) becomes

$$
\nabla_{\bar{l}}(\Delta s)=-2 k \sum_{i, j} G^{i j} \omega_{j \bar{k}} \nabla_{i} s,
$$

and we can take

$$
\frac{\partial s}{\partial t}=-\frac{\mathrm{i}}{2 k} \sum_{i, j} \nabla_{i}\left(G^{i j} \nabla_{j} s\right)
$$

as the heat equation defining the connection.

2. Although negative values of $k$ have not been considered here in the context of geometric quantization, note that putting $2 k=-\lambda$, Eq. (3.12) tells us that the second-order operator

$$
\sum_{i, j}\left(\nabla_{i}\left(G^{i j} \nabla_{j}\right)+2 \mathrm{i} G^{i j} \frac{\partial F}{\partial z_{i}} \nabla_{j}-\lambda f_{G}\right)
$$

is holomorphic. In the exact cohomology sequence of the sequence of sheaves

$$
0 \rightarrow \mathscr{D}^{1}\left(L^{-\lambda / 2}\right) \rightarrow \mathscr{D}^{2}\left(L^{-\lambda / 2}\right) \rightarrow S^{2} T \rightarrow 0,
$$


this is clear from $\delta(G)=0 \in H^{1}\left(M ; \mathscr{D}^{1}\left(L^{-\lambda / 2}\right)\right) \cong H^{1}(M ; T)$, but we have here an explicit differential geometric formula for this operator. The operators (one for each $G^{i j}$ ) act on $L^{-\lambda / 2} \cong K^{1 / 2}$ from (2.8). There are, however, no global sections of $K^{1 / 2}$, nor cohomology groups of higher degree, to get a global space on which the operators act.

We have now found in Theorem (3.6) a connection. We shall need the alternative Čech description of the coboundary map $\delta$ in order to proceed further.

First consider the Kodaira-Spencer class of a deformation of complex structure from the Čech point of view. We have a holomorphic fibration $Z \stackrel{p}{\longrightarrow} B$ over some open set $B \subset \mathbb{C}^{k}$ with coordinates $\left(t_{1}, \ldots, t_{k}\right)$ such that each fibre is a compact complex manifold $M_{t}^{n}$. With a suitable Stein covering of $Z$ we choose on each open set $U$ of the covering a coordinate system of the form

$$
\left(z_{1}, \ldots, z_{n}, t_{1}, \ldots, t_{k}\right)
$$

and consider the holomorphic vector fields $\frac{\partial}{\partial t_{A, \alpha}}$ on $U_{\alpha},(A=1, \ldots, k)$.

On an intersection $U_{\alpha} \cap U_{\beta}$, the vector field

$$
\frac{1}{i} \frac{\partial}{\partial t_{A, \alpha}}-\frac{1}{i} \frac{\partial}{\partial t_{A, \beta}}=X_{\alpha \beta}^{A}
$$

projects to zero in $B$ and hence is a Čech representative for a class in $H^{1}\left(M_{t} ; T\right)$ for each $t \in B$. This is the Kodaira-Spencer class for the infinitesimal deformation of complex structure of $M_{t}$ in the direction $t_{A}$.

If we additionally have a holomorphic line bundle $L$ on $Z$, inducing a family $\left(M_{t}, L_{t}\right)$ of complex structures and line bundles, then on each open set $U_{\alpha}$, sufficiently small, we can trivialize $L$ and then interpret $\frac{\partial}{\partial t_{A, \alpha}}$ as a first order differential operator on $L$ over $U_{\alpha}$. On the intersection $U_{\alpha} \cap U_{\beta}$, then $X_{\alpha \beta}$ in (3.15) from this point of view is a Čech cocycle for a class

$$
\left[X_{\alpha \beta}^{A}\right] \in H^{1}\left(M_{t}, \mathscr{D}^{1}(L)\right)
$$

whose symbol class is the Kodaira-Spencer class. This defines the deformation of holomorphic structure on $L$ in the direction $t_{A}$. (Note that different choices of $L$ on $Z$ are possible: $L$ and $L \otimes U$ for $U$ pulled back from the base $B$ define the same bundle on each fibre.)

Now consider the coboundary map (3.2)

$$
\delta: H^{0}\left(M ; S^{2} T\right) \rightarrow \mathbb{H}_{s}^{1}\left(M ; \mathscr{D}^{1}(L)\right)
$$

from the Čech point of view.

Given a global section $G$ of $S^{2} T$, we choose on each open set $M \cap U_{\alpha}$ of $M$ a holomorphic section $\Delta_{\alpha}$ of $\mathscr{D}^{2}(L)$ over $M \cap U_{\alpha}$, i.e. a holomorphic locally defined second order differential operator on $L$. On the intersection $M \cap U_{\alpha} \cap U_{\beta}, \Delta_{\alpha}-\Delta_{\beta}$ defines a section of $\mathscr{D}^{1}(L)$, since the principal symbols $G_{\alpha}, G_{\beta}$ agree as $G$ is globally defined. A Čech representative for $\delta(G)$ is then a pair of cocycles

$$
\left(\Delta_{\alpha}-\Delta_{\beta},-\Delta_{\alpha} s\right)
$$

in $C^{1}\left(M ; \mathscr{D}^{1}(L)\right) \oplus C^{0}(M ; L)[$ cf. (3.3)]. 
If we now have a holomorphic family of Kähler polarizations we can put the connection defined in Theorem (3.6) in Čech form. In fact, since $\frac{-\sigma \delta\left(G_{A}\right)}{(2 k+\lambda)} \in H^{1}(M ; T)$ is the Kodaira-Spencer class of the infinitesimal deformation in the direction $t_{A}$, with $G_{A}$ the corresponding symmetric tensor, then from (3.17) and (3.16),

$$
\frac{1}{i} \frac{\partial}{\partial t_{A, \alpha}}-\frac{1}{i} \frac{\partial}{\partial t_{A}, \beta}=-\frac{1}{(2 k+\lambda)}\left(\Delta_{A, \alpha}-\Delta_{A, \beta}\right)+D_{A, \beta}-D_{A, \alpha},
$$

where $D_{A, \alpha} \in C^{0}\left(M ; \mathscr{D}^{1}\left(L^{k}\right)\right)$ defines the coboundary $D_{A, \beta}-D_{A, \alpha}$. Thus

$$
\frac{\partial}{\partial t_{A, \alpha}}+\frac{i \Delta_{A, \alpha}}{(2 k+\lambda)}+i D_{A, \alpha}=\frac{\partial}{\partial t_{A, \beta}}+\frac{i \Delta_{A, \beta}}{(2 k+\lambda)}+i D_{A, \beta} \quad \text { on } \quad U_{\alpha} \cap U_{\beta},
$$

and we obtain a globally defined holomorphic heat operator

$$
\frac{\partial}{\partial t_{A}}+\frac{i \Delta_{A}}{(2 k+\lambda)}+i D_{A}=\frac{\partial}{\partial t_{A}}+P_{A}
$$

Note that neither the "Laplacian" part $P_{A}$ nor the "time derivative" $\frac{\partial}{\partial t_{A}}$ are globally defined but (3.18) shows that the combination $\frac{\partial}{\partial t_{A}}+P_{A}$ is indeed global. This heat equation is well-defined up to the addition of a constant since under hypothesis (i) of Theorem $(1.20), H^{0}\left(M ; \mathscr{D}^{1}\left(L^{k}\right)\right) \cong \mathbb{C}$. A covariant constant section of the vector bundle $V_{t}=H^{0}\left(M_{t}, L_{t}^{k}\right)$ (the direct image sheaf $\left.p_{*} L^{k}\right)$ over the family will be a solution of the heat equation (3.19). In any local coordinate system and local trivialisation of $L^{k}$, therefore, parallel translation along a holomorphic curve is given by solving an equation of the form

$$
\frac{d s}{d t}+\sum_{A} \frac{d t_{A}}{d t}\left(\sum_{i, j} G_{A}^{i j} \frac{\partial^{2} s}{\partial z_{i} \partial z_{j}}+\sum_{i} H_{A}^{i} \frac{\partial s}{\partial z_{i}}+K_{A} s\right)=0 .
$$

Since a holomorphic section of $L^{k}$ is determined by its value on any open set, this provides a local means of determining the connection. The same is true of the differential geometric heat equation (3.13), but there we always need local differential geometric information about the metric and curvature to write it down.

In both the Dolbeault and Čech descriptions of the connection, there is an indeterminacy in choice, reflecting the fact that the connection is really defined only on the projective space bundle $\mathbb{P}\left(H^{0}\left(M ; L^{k}\right)\right)$. This choice, and its dependence on $k$, is most explicitly given in the differential geometric formulation (3.11). There we considered the $(0,1)$ form

$$
\theta_{A}=\sum_{i, j}\left(2 \mathrm{i} G_{A}^{i j} \omega_{j l} \frac{\partial F}{\partial z_{i}}+\left(\nabla_{i} G_{A}^{i j}\right) \omega_{j l}\right),
$$

and chose a function $f_{A}$ such that

$$
\bar{\partial} f_{A}=\theta_{A} .
$$


If $f_{A}, f_{A}^{\prime}$ are two choices then $f_{A}-f_{A}^{\prime}$ is holomorphic and therefore a constant $c_{A}$. The heat equation (3.13) then changes by an additive constant

$$
\frac{i k c_{A}}{(2 k+\lambda)} \text {. }
$$

In the Čech formulation, this constant ambiguity appears in the choice of local holomorphic differential operator $D_{A, \alpha}$. In this context, we may regard $D_{A, \alpha}$ as varying holomorphically with respect to the base variable $t \in B$.

Making a choice of heat operator $\left(\frac{\partial}{\partial t_{A}}+P_{A}\right)$ over each open set $U_{\alpha}$ in a general complex base space, the difference on an intersection of two sets $U_{\alpha} \cap U_{\beta}$ defines a holomorphic function

$$
\frac{i k c_{A}(t)}{(2 k+\lambda)}
$$

on the intersection and hence a holomorphic 1-form

$$
\phi_{k}=\sum_{A} \frac{i k c_{A} d t_{A}}{(2 k+\lambda)}
$$

which represents a Čech cohomology class in $H^{1}\left(B ; T_{B}^{*}\right)$. If this class were to vanish, then by judicious choices of $f_{A}$, we could find a well-defined holomorphic connection on the vector bundle $V$. This obstruction is called the central charge, since if we had a connection with central curvature on $V$, then the curvature would represent the same class [at least if $B$ was a compact Kähler manifold so that $\left.H^{1}\left(B ; T^{*}\right) \cong H^{1,1}(B ; \mathbb{C})\right]$.

Thus far, we have said little about the curvature of the connection defined in Theorem (3.6). Note that the holomorphic description by Čech cohomology shows that for a holomorphic family the connection itself is holomorphic and thus the curvature is of type $(2,0)$ - the $(1,1)$ and $(0,2)$ components already vanish. Note also that to prove flatness it is sufficient to consider holomorphic families since the deformations we are considering arise from deformations of conformal structure on $\Sigma$ and these are holomorphically parametrized by a complex manifold Teichmüller space.

In the next section we shall prove flatness by using the Čech formalism, and some basic facts about symmetric tensors $G \in H^{0}\left(M ; S^{2} T\right)$ on $M$.

\section{Integrable Systems and Flatness of the Connection}

The symmetric tensors $G \in H^{0}\left(M ; S^{2} T\right)$ which yield the infinitesimal deformations of Kähler polarizations were defined in (2.15) by

$$
G(\alpha, \alpha)=\int_{\Sigma} \operatorname{Tr} \alpha^{2} *
$$

where $\alpha \in H^{0}\left(\Sigma ; \operatorname{End}_{0} E \otimes K\right)$ is considered as a cotangent vector to $M$, $\operatorname{Tr} \alpha^{2} \in H^{0}\left(\Sigma ; K^{2}\right)$ is a quadratic differential and $* \in \Omega^{0,1}\left(\Sigma ; K^{-1}\right)$ is a deformation of conformal structure. 
This definition represents $G$ explicitly as a holomorphic function on the cotangent bundle $T^{*} M$ which is homogeneous of degree 2 in the fibre directions. We may define more functions of this type of homogeneity $l$ simply by taking $\beta \in \Omega^{0,1}\left(\Sigma ; K^{-l-1}\right)$ and defining

$$
H(\alpha)=\int_{\Sigma} \operatorname{Tr}\left(\alpha^{l}\right) \beta .
$$

Proposition (4.2) (see [7]). With respect to the canonical holomorphic symplectic structure on $T^{*} M$, any two functions $H_{1}, H_{2}$ of the form (4.1) Poisson-commute.

Proof. This is a very general fact. Following [7], we prove it by appealing to infinite-dimensional symplectic geometry. As in Sect. 2 we consider $\mathscr{A}$ to be the complex affine space of all Cauchy-Riemann operators on a $C^{\infty}$ vector bundle $E$, with an action of the group $\mathscr{G}^{c}$ of $C^{\infty}$ automorphisms. This action lifts to a symplectic action on the cotangent bundle

$$
T^{*} \mathscr{A} \cong \mathscr{A} \times \Omega^{1,0}\left(\Sigma ; \operatorname{End}_{0} E\right) .
$$

A point of $T^{*} \mathscr{A}$ consists of a pair $\left(\bar{\partial}_{A}, \alpha\right)$ and the function $H$ in (4.1) is clearly welldefined as a function on $T^{*} \mathscr{A}$, independent of the $\mathscr{A}$-variables. Since the $\mathscr{A}$-variables and $\Omega^{1,0}\left(\Sigma ; \operatorname{End}_{0} E\right)$-variables are conjugate with respect to the canonical symplectic form, the two functions $H_{1}$ and $H_{2}$ certainly Poissoncommute on $T^{*} \mathscr{A}$.

Now the moment map for the action of $\mathscr{G}^{c}$ is

$$
\mu\left(\bar{\partial}_{A}, \alpha\right)=\bar{\partial}_{A} \alpha \in \Omega^{1,1}\left(\Sigma ; \operatorname{End}_{0} E\right) \cong\left(\operatorname{Lie}\left(\mathscr{G}^{c}\right)\right)^{*},
$$

so the Marsden-Weinstein quotient (here in the complex case) consists of the equivalence classes of pairs $\left(\bar{\partial}_{A}, \alpha\right)$ such that $\bar{\partial}_{A} \alpha=0$, i.e. $\alpha \in H^{0}\left(\Sigma ; \operatorname{End}_{0} E \otimes K\right)$. If we restrict to the stable holomorphic structures $\bar{\partial}_{A}$ on $E$, then this is just the cotangent bundle $T^{*} M$ of the moduli space of stable bundles.

Now $H_{1}$ and $H_{2}$ are invariant under the action of $\mathscr{G}^{c}$, since it acts on $\alpha$ by conjugation and $\operatorname{Tr}\left(\alpha^{l}\right)$ is conjugation-invariant. Hence being invariant under $\mathscr{G}^{c}$ and Poisson-commuting, they inherit the same property on the quotient $T^{*} M$, where they are of course holomorphic.

In the above proposition we restricted attention to stable complex structures, but in fact there is a notion of stability for a pair of objects - a holomorphic vector bundle $E$ of rank $m$ over $\Sigma$ and a Higgs field $\alpha \in H^{0}\left(\Sigma ; \operatorname{End}_{0} E \otimes K\right)$. This definition is the standard stability criterion for subbundles $U C E$ as in Sect. 2, but restricted only to those $U$ which are $\alpha$-invariant. (In [6], which deals with the rank 2 case these are called "stable pairs." In [18], which holds for general rank - and dimension of the base $\Sigma$ - they are called stable "Higgs bundles.") There is a good moduli space for these Higgs bundles (see $[6,18,13]$ ) denoted by $\mathscr{M}^{2 n}$ which contains $T^{*} M^{n}$ as an open set and is a (non-compact) complex symplectic manifold, extending the canonical symplectic structure on $T^{*} M$. Functions of type (4.1) are well-defined on $\mathscr{M}$ and Poisson-commute there. It follows from [6, 7, and 18] (see also [3]) that these holomorphic functions make $\mathscr{M}$ into a completely integrable Hamiltonian system. More precisely:

Proposition (4.3). Let $V=\bigoplus_{i=2}^{m} H^{0}\left(\Sigma ; K^{i}\right)$, and define $p: \mathscr{M}^{2 n} \rightarrow V$ by

$$
p\left(\bar{\partial}_{A}, \alpha\right)=\left(\operatorname{Tr} \alpha^{2}, \operatorname{Tr} \alpha^{3}, \ldots, \operatorname{Tr} \alpha^{m}\right),
$$


then

i) $p$ is proper,

ii) $\operatorname{dim} \mathscr{M}=2 \operatorname{dim} V=2 n$,

iii) the $n$ functions $p_{i}$ defined by $p$ Poisson-commute,

iv) the generic fibre of $p$ is an abelian variety.

These properties of the moduli space $\mathscr{M}$ allow us to deduce the following property of the vector space of holomorphic tensors on $M$, the moduli space we are primarily interested in:

Proposition (4.4). The linear map

$$
f: H^{1}\left(\Sigma ; K^{-1}\right) \rightarrow H^{0}\left(M ; S^{2} T\right)
$$

defined by

$$
f(\beta)(\alpha, \alpha)=\int_{\Sigma}\left(\operatorname{Tr} \alpha^{2}\right) \beta
$$

for $\alpha \in H^{0}\left(\Sigma ;\right.$ End $\left._{0} E \otimes K\right)$ and $\beta \in \Omega^{0,1}\left(\Sigma ; K^{-1}\right)$ is an isomorphism.

Proof. The method of proof is to take $G \in H^{0}\left(M ; S^{2} T\right)$ and think of it as a holomorphic function of degree 2 on the cotangent bundle $T^{*} M$. We then want to extend it to a holomorphic function on $\mathscr{M}$ by Hartog's theorem. To do this we should prove that the codimension of the complement of $T^{*} M$ in $\mathscr{M}$ is greater than one.

Recall that (see [2]) every holomorphic bundle $E$ has a canonical filtration

$$
0=E_{0} \subset E_{1} \subset \ldots \subset E_{r}=E
$$

by bundles for which $D_{i}=E_{i} / E_{i-1}$ is semi-stable and

$$
\frac{\operatorname{deg} D_{1}}{\operatorname{rk} D_{1}}>\frac{\operatorname{deg} D_{2}}{\operatorname{rk} D_{2}}>\ldots>\frac{\operatorname{deg} D_{r}}{\operatorname{rk} D_{r}} .
$$

This type determines a stratification of the space of holomorphic structures on $E$, whose maximal stratum consists of the stable structures. There is a natural partial ordering on the strata (cf. [2]) which shows that the maximal strata in the complement of the stable structures consist of holomorphic structures which are extensions

$$
0 \rightarrow U_{1} \rightarrow E \rightarrow U_{2} \rightarrow 0
$$

where $\mu\left(U_{1}\right)>\mu\left(U_{2}\right)(\mu(U)=\operatorname{deg} U / \operatorname{rk} U)$ and $U_{1}$ and $U_{2}$ are stable. For stable bundles it is a standard fact that if $\mu(U)>\mu(V)$ then there are no non-trivial homomorphisms from $U$ to $V$, and it follows from this that $E$ is uniquely expressible as an extension in this way.

The stratification induces one on the Higgs bundle moduli space $\mathscr{M}$, the maximal stratum being $T^{*} M$. We wish to estimate the dimension of the space of stable Higgs bundles of type (4.5). First note that in order for $E$ to appear as a stable Higgs bundle, there must exist a Higgs field $\alpha$ for which $U_{1}$ is not invariant. In particular, $H^{0}\left(\Sigma ; U_{1}^{*} \otimes U_{2} \otimes K\right) \neq 0$. Conversely, if this holds, it is easy to construct a stable Higgs field on $U_{1} \oplus U_{2}$. This condition on $U_{1}$ and $U_{2}$ is therefore necessary 
and sufficient to determine the relevant extensions. We may remark that by Serre duality $H^{1}\left(\Sigma ; U_{2}^{*} \otimes U_{1}\right) \neq 0$ so that non-trivial extensions exist.

Let $\operatorname{deg} U_{i}=k_{i}$ and $\operatorname{rk} U_{i}=n_{i}$. Since $U_{i}$ is stable its holomorphic structure is parametrized by a moduli space of dimension $n_{i}^{2}(g-1)+1$. We are fixing the holomorphic structure on $\Lambda^{m} E$, which yields the number of parameters for choosing $U_{1}$ and $U_{2}$ as

$$
n_{1}^{2}(g-1)+n_{2}^{2}(g-1)+2-g .
$$

For fixed $U_{1}$ and $U_{2}$, the holomorphic structures $E$ expressed as extensions (4.5) are parametrized by $\mathbb{P}\left(H^{1}\left(\Sigma ; U_{2}^{*} \otimes U_{1}\right)\right)$, so we have an additional number of parameters

$$
\operatorname{dim} H^{1}\left(\Sigma ; U_{2}^{*} \otimes U_{1}\right)-1 .
$$

Finally, stability of the Higgs bundle implies that the automorphisms of $E$ act freely on the stable Higgs fields, so for each choice of holomorphic structure on $E$, the number of extra parameters is $\operatorname{dim} H^{0}\left(\Sigma ; \operatorname{End}_{0} E \otimes K\right)-\operatorname{dim} H^{0}\left(\Sigma ; \operatorname{End}_{0} E\right)$ which by Riemann-Roch and Serre duality is

$$
\left(\left(n_{1}+n_{2}\right)^{2}-1\right)(g-1) \text {. }
$$

The dimension of $\mathscr{M}$ is $2\left(\left(n_{1}+n_{2}\right)^{2}-1\right)(g-1)$, so from the above expressions the codimension of the stratum we are considering is

$$
c=2 n_{1} n_{2}(g-1)-\operatorname{dim} H^{1}\left(\Sigma ; U_{2}^{*} \otimes U_{1}\right) .
$$

To estimate $c$ we use two methods. For the first, we choose a positive integer $d$ such that

$$
d>\frac{k_{1}}{n_{1}}-\frac{k_{2}}{n_{2}}>0 .
$$

Let $L$ be a line bundle of degree $d$ with a section $s$. The inequality above implies $\mu\left(U_{2} \otimes L\right)>\mu\left(U_{1}\right)$ and hence $H^{0}\left(\Sigma ; U_{2}^{*} \otimes U_{1} \otimes L^{*}\right)=0$. By Riemann-Roch

$$
\operatorname{dim} H^{1}\left(\Sigma ; U_{2}^{*} \otimes U_{1} \otimes L^{*}\right)=k_{2} n_{1}-k_{1} n_{2}+n_{1} n_{2}(d+g-1) .
$$

Tensoring with the section $s$ gives a surjective map of this space to $H^{1}\left(\Sigma ; U_{2}^{*} \otimes U_{1}\right)$, so

$$
\operatorname{dim} H^{1}\left(\Sigma ; U_{2}^{*} \otimes U_{1}\right) \leqq k_{2} n_{1}-k_{1} n_{2}+n_{1} n_{2}(d+g-1) .
$$

Now if $d$ is the smallest positive integer with the property above, then

$$
d-1 \leqq \frac{k_{1}}{n_{1}}-\frac{k_{2}}{n_{2}}
$$

and it follows that $\operatorname{dim} H^{1}\left(\Sigma ; U_{2}^{*} \otimes U_{1}\right) \leqq n_{1} n_{2} g$. Hence from (4.6) we obtain

$$
c \geqq n_{1} n_{2}(g-2) \text {. }
$$

This inequality establishes that the codimension is at least 2 if $g>3$ or if $g=3$ and $\operatorname{rk} E>2$ but not for $g=2$ or $g=3$ and $\operatorname{rk} E=2$. To deal with these cases we use a second argument. 
Consider a holomorphic section $u$ of $K$ and the exact sequence of sheaves:

$$
0 \rightarrow \mathcal{O}_{\Sigma}\left(U_{2}^{*} \otimes U_{1}\right) \stackrel{u}{\longrightarrow} \mathcal{O}_{\Sigma}\left(U_{2}^{*} \otimes U_{1} \otimes K\right) \rightarrow \mathcal{O}_{D}\left(U_{2}^{*} \otimes U_{1} \otimes K\right) \rightarrow 0,
$$

where $D$ is the divisor of $u$, of degree $2 g-2=\operatorname{deg} K$.

Since $\mu\left(U_{1}\right)>\mu\left(U_{2}\right), H^{0}\left(\Sigma ; U_{1}^{*} \otimes U_{2}\right)$ and its Serre dual $H^{1}\left(\Sigma ; U_{1}^{*} \otimes U_{1} \otimes K\right)$ vanish, so from the exact sequence of cohomology groups

$$
\begin{aligned}
& \operatorname{dim} H^{0}\left(\Sigma ; U_{2}^{*} \otimes U_{1} \otimes K\right)-\operatorname{dim} H^{0}\left(\Sigma ; U_{2}^{*} \otimes U_{1}\right) \\
& \quad=(2 g-2) n_{1} n_{2}-\operatorname{dim} H^{1}\left(\Sigma ; U_{2}^{*} \otimes U_{1}\right)=c .
\end{aligned}
$$

Now sections of vector bundles can be interpreted as sections of line bundles: $H^{0}\left(\Sigma ; U_{2}^{*} \otimes U_{1}\right)=H^{0}(P ; U)$ where $P$ is the projective bundle of $U_{2}^{*} \otimes U_{1}^{*}$ and $U$ the hyperplane bundle along the fibres. Consider the map

$$
\mathbb{P}\left(H^{0}(P ; U)\right) \times \mathbb{P}\left(H^{0}(P ; K)\right) \rightarrow \mathbb{P}\left(H^{0}(P ; U \otimes K)\right)
$$

obtained by adding effective divisors. It is finite-to-one, so

$$
\operatorname{dim} H^{0}(P ; U)-1+\operatorname{dim} H^{0}(P ; K)-1 \leqq \operatorname{dim} H^{0}(P ; U \otimes K)-1
$$

and hence

$$
c=\operatorname{dim} H^{0}\left(\Sigma ; U_{2}^{*} \otimes U_{1} \otimes K\right)-\operatorname{dim} H^{0}\left(\Sigma ; U_{2}^{*} \otimes U_{1}\right) \geqq g-1
$$

Here, if equality holds, every divisor of the system $U \otimes K$ on $P$ is reducible. Applying Bertini's theorem (after removing fixed components) this can only hold if

$$
\operatorname{dim} H^{0}\left(\Sigma ; U_{2}^{*} \otimes U_{1} \otimes K\right) \leqq 2 .
$$

But by Riemann-Roch this gives

$$
n_{1} n_{2}(g-1)+k_{1} n_{2}-k_{2} n_{1} \leqq 2 .
$$

Since $k_{1} n_{2}-k_{2} n_{1}>0$, the only possibility for equality is $g=2, \operatorname{rk} E=2$. Excluding this case, the codimension $c$ is greater than or equal to 2 .

We can, therefore, apply Hartog's theorem and extend $G$ to a holomorphic function on $\mathscr{M}$, homogeneous of degree 2 with respect to the $\mathbb{C}^{*}$-action

$$
\left(\bar{\partial}_{A}, \alpha\right) \rightarrow\left(\bar{\partial}_{A}, \lambda \alpha\right) \text {. }
$$

Now we use the integrable system of (4.3) - $G$ is constant on each compact fibre of $p$ and hence is the pull-back of a holomorphic function on $V$, homogeneous of degree 2 . However, the weights of the $\mathbb{C}^{*}$-action on $V$ are $(2,3,4, \ldots, m)$ so the only holomorphic functions of degree 2 are of the form $g \circ p$ for $g \in H^{0}\left(\Sigma ; K^{2}\right)^{*}$ $=H^{1}\left(\Sigma ; K^{-1}\right)$, i.e. the functions of degree 2 referred to in the proposition.

This shows that $f$ is surjective. The functional independence of these functions certainly implies their linear independence so $f$ is indeed an isomorphism.

(Note that we can prove (2.9) in the same way - a global section of $T$ will define a holomorphic function on $V$ of homogeneity one which is impossible since 2 is the smallest weight.)

Propositions (4.2) and (4.4) now allow us to prove the required flatness. 
Theorem (4.9). The connection defined in Theorem (3.6) is flat.

Proof. We adopt the Čech point of view and consider the holomorphic heat operators $\frac{\partial}{\partial t_{A}}+P_{A}$ and $\frac{\partial}{\partial t_{B}}+P_{B}$ and their commutator

$$
\left[\frac{\partial}{\partial t_{A}}+P_{A}, \frac{\partial}{\partial t_{B}}+P_{B}\right]
$$

This is a globally defined operator on $L^{k}$ which involves no $t$-differentiation. We can write it locally in the form

$$
\frac{\partial P_{B}}{\partial t_{A}}-\frac{\partial P_{A}}{\partial t_{B}}+\left[P_{A}, P_{B}\right]
$$

Now $P_{A}$ and $P_{B}$ are second order operators, and so therefore is the first term $\frac{\partial P_{B}}{\partial t_{A}}-\frac{\partial P_{A}}{\partial t_{B}}$. The second term is a 3 rd-order operator whose principal symbol, thought of as a function homogeneous of degree 3 on $T^{*} M$, is the Poisson bracket of the symbols of $P_{A}$ and $P_{B}$ (see e.g. [5]).

However, from Proposition (4.2) these functions Poisson-commute and so (4.10) is a globally defined 2 nd order operator on $L^{k}$, i.e. for each $t$, a holomorphic section of $\mathscr{D}^{2}\left(L^{k}\right)$ on $M_{t}$, with symbol $G$.

Now consider the exact sequence of sheaves

$$
0 \rightarrow \mathscr{D}^{1}\left(L^{k}\right) \rightarrow \mathscr{D}^{2}\left(L^{k}\right) \rightarrow S^{2} T \rightarrow 0,
$$

and the exact sequence of cohomology groups

$$
0 \rightarrow H^{0}\left(M ; \mathscr{D}^{1}\left(L^{k}\right)\right) \rightarrow H^{0}\left(M ; \mathscr{D}^{2}\left(L^{k}\right)\right) \rightarrow H^{0}\left(M ; S^{2} T\right) \stackrel{\delta}{\longrightarrow} H^{1}\left(M ; \mathscr{D}^{1}\left(L^{k}\right)\right) \rightarrow \ldots
$$

From Proposition (4.4) $G$ arises from some deformation of conformal structure on $\Sigma$ and from Theorem (3.6), $\delta(G)$ is a non-zero multiple of the Kodaira-Spencer map of this deformation. Also, from (2.11), the Kodaira-Spencer map is injective hence

$$
\sigma \delta: H^{0}\left(M ; S^{2} T\right) \rightarrow H^{1}(M ; T)
$$

is an isomorphism from (4.4).

In particular $\delta$ itself is injective. Thus from (4.11) the holomorphic section of $\mathscr{D}^{2}\left(L^{k}\right)$ is actually a section of $\mathscr{D}^{1}\left(L^{k}\right)$-a first order operator. But now the vanishing of $H^{0}(M ; T)$ and compactness of $M$ show that it must be a constant. Hence

$$
\left[\frac{\partial}{\partial t_{A}}+P_{A}, \frac{\partial}{\partial t_{B}}+P_{B}\right]
$$

is a constant $C_{A B}$.

Now from the definition of the connection, parallel translation of a global section of $L^{k}$ along holomorphic curves in the $t_{A}$-direction means solving the holomorphic heat equation

$$
\frac{\partial s}{\partial t_{A}}+P_{A} s=0
$$


Since the operators $\frac{\partial}{\partial t_{A}}+P_{A}, \frac{\partial}{\partial t_{B}}+P_{B}$ commute up to a constant, then it is clear that, for given choices of $P_{A}$ and $P_{B}$, the curvature of the connection is a scalar, and hence the projective space connection is flat.

Remarks (4.12). 1. In the abelian situation, the most natural choice of local coordinates and trivialization of $L^{k}$ is to pass to the universal covering $\mathbb{C}^{g}=H^{1}(\Sigma ; \mathcal{O})$ of the torus $H^{1}(\Sigma ; \mathcal{O}) / H^{1}(\Sigma ; \mathbb{Z})$. On $\mathbb{C}^{g}$, the global symmetric tensors are just constant symmetric matrices. In this situation Proposition (4.4) does not hold - not all principally polarized abelian varieties are Jacobians, but it is true that every symmetric tensor gives a deformation of a Kähler polarization of the torus. Indeed, the symmetric tensors are the tangent space of the Siegel upper half space which parametrizes all principally polarized abelian varieties. The holomorphic heat operator in this trivialization is

$$
\frac{\partial}{\partial T_{i j}}+\frac{i}{4 \pi k} \frac{\partial^{2}}{\partial z_{i} \partial z_{j}}
$$

which has as global solutions the classical theta functions of level $k$

$$
\theta_{m}=\sum_{\substack{l \in \mathbb{Z}^{n} \\ l \equiv m(k)}} e^{\pi i / k\langle l, T l\rangle} e^{2 \pi i\langle l, z\rangle} .
$$

Thus the theta functions are the covariant constant sections of the flat connection. The flatness of the connection could in fact be proved a priori by the arguments of Theorem (4.9), using the elementary fact that

$$
H^{0}\left(M ; S^{2} T\right) \stackrel{\delta}{\longrightarrow} H^{1}(M ; T)
$$

is injective for an abelian variety.

2. Note that the proof of (4.9) concerned primarily the heat operator, before it was applied to any global section. Recall from Remark (3.14) that when $2 k=-\lambda$, there is no heat operator, but instead a global holomorphic second order operator $\Delta_{G}$ acting on $K^{1 / 2}$, with symbol $G \in H^{0}\left(M ; S^{2} T\right)$. We have seen in Proposition (4.2) that the symbols of $\Delta_{G}$ and $\Delta_{H}$ commute and so $\left[\Delta_{G}, \Delta_{H}\right]$ is a second order operator. We may show in fact that this vanishes - the operators themselves commute.

The key to this is to note that for any operator $D$ on $K^{1 / 2}$ there is a formal holomorphic adjoint operator $D^{*}$, a canonically defined operator such that for any local sections $s, t$ of $K^{1 / 2}$ on an open set $U \subset M$,

$$
(D s) t-s\left(D^{*} t\right)=\partial \phi(s, t) \in H^{0}(U ; K) \text {. }
$$

Here $\phi(s, t)$ is a holomorphic $(n-1)$-form, so $\partial \phi$ is a holomorphic $n$-form - a local section of $K$. If $D$ is of order $m$, with symbol $G$, then the symbol of $D^{*}$ is $(-1)^{m} G$.

Hence $\Delta_{G}-\Delta_{G}^{*}$ is a holomorphic 1 st-order operator on $K^{1 / 2}$ but we know since $H^{0}(M ; T)=0$ that this is just a constant scalar $c$. Since $\Delta_{G}-\Delta_{G}^{*}$ is formally skewadjoint it follows that $c=-c$, i.e. $c=0$.

Hence each $\Delta_{G}$ is self-adjoint. This means that the commutator $\left[\Delta_{G}, \Delta_{H}\right]$ is skew-adjoint. However, since the symbols Poisson-commute it is 2 nd order with symbol $F \in H^{0}\left(M ; S^{2} T\right)$. Being skew-adjoint $F=-F=0$, so the commutator is 
first order and hence constant. Then, as above, skew-adjointness implies the constant vanishes.

Thus

$$
\Delta_{G} \Delta_{H}=\Delta_{H} \Delta_{G} .
$$

If we set $W=H^{0}\left(M ; \mathscr{D}^{2}\left(K^{1 / 2}\right)\right)$, the vector space of these operators, then the symbol map expresses $W$ as an extension

$$
0 \rightarrow \mathbb{C} \rightarrow W \stackrel{\sigma}{\longrightarrow} H^{0}\left(M ; S^{2} T\right) \rightarrow 0
$$

(any two such operators with the same symbol differ by a constant). From Proposition (4.4), $H^{0}\left(M ; S^{2} T\right)$ is canonically isomorphic to $H^{1}\left(\Sigma ; K^{-1}\right)$ - the tangent space to Teichmüller space. Thus, as the conformal structure on $\Sigma$ varies, $W$ defines a vector bundle over Teichmüller space which is an extension

$$
0 \rightarrow \mathcal{O} \rightarrow W \rightarrow T \rightarrow 0
$$

and hence defines, on any family $B$ of conformal structures an extension class in $H^{1}\left(B ; T_{B}^{*}\right)$. This is essentially the central charge class $-i \lambda \sum_{A} c_{A} d t_{A}$ [cf. (3.21)].

\section{Singular Moduli Spaces}

Let us finally outline how to deal with the case where the moduli space $M$ is singular. From the point of view of flat connections the singularities are the connections which are reducible, i.e. $E=E_{1} \oplus E_{2}$, where $E_{1}$ and $E_{2}$ are preserved by the flat connection. In the holomorphic viewpoint they are the $S$-equivalence classes of semi-stable bundles - a notion of equivalence which is weaker than holomorphic equivalence. The moduli space $M$ is a normal projective variety [12]. Normality means in particular that there is a Hartog's theorem for extending holomorphic sections of line bundles from the open stable subset $M^{s} \subset M$ to $M$. Since $M^{s}$ is a manifold, and our description of the connection in either the Dolbeault or Čech viewpoint is local then we can hope to work on the noncompact manifold $M^{s}$ and rely on Hartog's theorem to extend to $M$ [and give in particular finite dimensionality of $\left.H^{0}\left(M ; L^{k}\right)\right]$.

There are a number of occasions where we have used smoothness and compactness. We list here the properties we require of $M^{s}$ to make our arguments carry through:

i) $H^{0}\left(M^{s} ; \mathcal{O}\right) \cong \mathbb{C}$,

ii) $H^{0}\left(M^{s} ; T\right)=0$,

iii) $H^{1}\left(M^{s} ; \mathcal{O}\right)=0$,

iv) $H^{1}\left(\Sigma ; K^{-1}\right) \rightarrow H^{0}\left(M^{s} ; S^{2} T\right)$ is an isomorphism,

v) the Kodaira-Spencer map $H^{1}\left(\Sigma ; K^{-1}\right) \rightarrow H^{1}\left(M^{s}, T\right)$ is injective,

vi) the dimension of $H^{0}\left(M^{s} ; L^{k}\right)$ is a deformation invariant for the standard family of Kähler polarisations.

Property (i) is immediate from Hartog's theorems and the compactness of $M$. Properties (ii) and (iv) follow similarly by imitating the argument of Proposition (4.4) with $\mathscr{M}$ replaced by the corresponding singular space for semi-stable Higgs bundles. This is a normal, quasi-projective variety [13] so Hartog's theorem 
can be applied to the holomorphic functions of homogeneity 1 and 2 respectively defined on the smooth manifold $T^{*} M^{s} \subset \mathscr{M}$ by sections of $T$ and $S^{2} T$ on $M^{s}$. Here the arguments of (4.4) can be applied again, though taking account also of the semistable case $\mu\left(U_{1}\right)=\mu\left(U_{2}\right)$. The case $g=2, \operatorname{rk} E=2$ is indeed special - the moduli space $M$ is $\mathbb{C} P^{3}$ which clearly does have holomorphic vector fields.

Properties (iii) and (v) involve 1st cohomology groups rather than zeroth groups. For this a general Hartog's-type theorem exists (see [16]) for the complements of analytic sets of codimension $>2$. We follow the same procedure as in Sect. 4 (cf. [7]). By contraction a class in $H^{1}\left(M^{s} ; S^{k} T\right)$ defines a cohomology class in $H^{1}\left(T^{*} M^{s} ; \mathcal{O}\right)$ of homogeneity $k$. The Leray spectral sequence for the projective bundle $\mathbb{P}\left(T^{*} M^{s}\right)$ shows that the space of classes in $H^{1}\left(T_{0}^{*} M^{s} ; \mathcal{O}\right)$ (where $T_{0}^{*} M^{s}$ is the complement of the zero section) of homogeneity $k$ correspond bijectively to $H^{1}\left(M^{s} ; S^{k} T\right)$. The Hartog-type extension theorem shows that the classes in $T^{*} M^{s}$ are determined by their restriction to $T_{0}^{*} M^{s}$ since $\operatorname{dim} M^{s}>1$. We may therefore consider $H^{1}\left(M^{s} ; \mathcal{O}\right)$ and $H^{1}\left(M^{s} ; T\right)$ as the subspaces of $H^{1}\left(T^{*} M^{s} ; \mathcal{O}\right)$ of homogeneity 0 and 1 respectively.

To extend to $\mathscr{M}$, we have to extend across the singularities and the strata of the Harder-Narasimhan filtration. The expression (4.8) is greater than 2 if $g>2$.

Apart from this case then, the classes can be extended. The singularities of $\mathscr{M}$ are represented by reducible Higgs bundles. Since the dimension of a Higgs moduli space is twice that of the corresponding stable bundle moduli space, the codimension of the singular set of $\mathscr{M}$ is twice that of $M$, and hence by normality of $M$ at least 4 . Hence these classes extend to classes in $H^{1}(\mathscr{M} ; \mathcal{O})$.

We now apply the proper map $\phi: \mathscr{M} \rightarrow V$ of Proposition (4.3). The higher cohomology groups of the vector space $V$ vanish, so

$$
H^{1}(\mathscr{M} ; \mathcal{O}) \cong H^{0}\left(V ; R_{p^{*}}^{1} \mathcal{O}\right),
$$

where $R_{p^{*}}^{1} \mathcal{O}$ is the 1 st direct image sheaf.

We shall use the following result to identify classes in $H^{1}(\mathscr{M}, \mathcal{O})$ :

Proposition (5.2). There is a natural isomorphism

$$
\Psi: H^{0}(V ; \mathcal{O}) \otimes V^{*} \cong H^{1}(\mathscr{M} ; \mathcal{O})
$$

such that $\Psi(\varrho(\lambda) f)=\lambda^{-1} \sigma(\lambda) \Psi(f)$ for the natural actions, $\varrho, \sigma$ of $\lambda \in \mathbb{C}^{*}$ on both sides.

Proof. To define $\Psi$, note that every linear functional $f_{i} \in V^{*}$ defines a Hamiltonian function $f_{i} \circ p$ on $\mathscr{M}^{s}$ whose corresponding vector field $X_{i}$ is tangent to the fibres of p. Also $\mathscr{M}^{s}$ carries a natural Kähler form $\omega$ (see [6]) which restricts to the symplectic form on $M^{s} \subset T M^{s} \subset \mathscr{M}^{s}$. This defines a class $[\omega] \in H^{1}\left(\mathscr{M}^{s} ; T_{\mathscr{M}}^{*}\right)$ which gives a homomorphism

$$
\phi: H^{0}\left(\mathscr{M}^{s} ; T_{\mathcal{M}}\right) \rightarrow H^{1}\left(\mathscr{M}^{s} ; \mathcal{O}\right) \cong H^{1}(\mathscr{M} ; \mathcal{O}) .
$$

Restricting $\phi$ to the vector fields $X_{i}$ gives a map

$$
V^{*} \rightarrow H^{1}(\mathscr{M} ; \mathcal{O})
$$

which extends obviously to define the $H^{0}(V ; \mathcal{O})$ - module map $\Psi$. The homogeneity of $\Psi$ follows from the fact that the holomorphic symplectic form on the cotangent bundle $T^{*} M^{s}$ has homogeneity 1 . 
We use (5.1) to prove that $\Psi$ is an isomorphism. Firstly, recall that the point $p(A, \Phi)$ for $(A, \Phi) \in \mathscr{M}$ is given by $\left(a_{2}, a_{3}, \ldots, a_{m}\right)$ where $a_{i} \in H^{0}\left(\Sigma ; K^{i}\right)$. This defines a spectral curve (see [11])

$$
\operatorname{det}(\eta+\Phi)=\eta^{m}+a_{2} \eta^{m-2}+\ldots+a_{m}=0
$$

in the surface which is the total space of the cotangent bundle of $\Sigma$.

On the spectral curve $\hat{\Sigma}, \Phi$ has a single-valued eigenvalue $-\eta$ and the kernel of $\eta+\Phi$ defines a rank 1 torsion-free sheaf on $\hat{\Sigma}$, at least if $\hat{\Sigma}$ is stable, for example if it is irreducible and has only nodes as singularities. The fibre of $p$ is then the space of equivalence classes of such sheaves. If $\hat{\Sigma}$ is non-singular this is essentially the Jacobian of $\hat{\Sigma}$. When $\hat{\Sigma}$ is stable this is a stable quasi-abelian variety $X$ (see $[14,10]$ ) and in particular

$$
\operatorname{dim} H^{1}\left(X ; \mathcal{O}_{X}\right)=\operatorname{dim} X .
$$

This means that the sheaf $R_{p^{*}}^{1} \mathcal{O}$ is a holomorphic vector bundle over the open set in $V$ of $\left(a_{2}, \ldots, a_{m}\right)$ which define stable curves. In fact, apart from the ubiquitous special case $g=2, m=2$, a simple application of Bertini's theorem shows that the spectral curves with just one node or less form the complement of an analytic set $D \subset V$ whose codimension is greater than one. Since by Hartog's theorem classes in $H^{1}(\mathscr{M} ; \mathcal{O})$ are determined by their restriction to complements of codimension 2 sets, (5.1) gives

$$
H^{1}(\mathscr{M} ; \mathcal{O}) \cong H^{0}\left(V \backslash D ; R_{p^{*}}^{1} \mathcal{O}\right)
$$

Now for a non-singular abelian variety $X$ the cup-product with a Kähler class $[\omega] \in H^{1}\left(X ; T^{*}\right)$ gives an isomorphism

$$
H^{0}(X ; T) \stackrel{[\omega]}{\longrightarrow} H^{1}(X ; \mathcal{O}) .
$$

The same is certainly true for the singular abelian variety corresponding to a spectral curve with one node - the question reduces to checking the case of the quasi-abelian variety which is a rational curve with a node. Now since the vector fields $X_{1}, \ldots, X_{n}$ form a basis for $H^{0}\left(X ; T_{X}\right)=\operatorname{Ext}^{0}\left(\Omega_{X}^{1}, \mathcal{O}_{X}\right)$ we see that over $V \backslash D$, the vector bundle $R_{p^{*}}^{1} \mathcal{O}$ is isomorphic with the trivial bundle $V \backslash D \times V^{*}$ by the map $\phi$. From (5.4) we have, as required,

$$
\begin{aligned}
H^{1}(\mathscr{M} ; \mathcal{O}) & \cong H^{0}(V \backslash D ; \mathcal{O}) \otimes V^{*} \\
& \cong H^{0}(V ; \mathcal{O}) \otimes V^{*} \text { by Hartog's theorem. }
\end{aligned}
$$

From Proposition (5.2) we may easily deduce property (iii), that $H^{1}\left(M^{s}, \mathcal{O}\right)=0$. Indeed, a class of homogeneity zero in $H^{1}(\mathscr{M} ; \mathcal{O})$ corresponds under $\Psi$ to a class of negative homogeneity in $H^{0}(V ; \mathcal{O}) \otimes V^{*}$, and there are no such holomorphic functions with values in $V^{*}$. There is only one subspace of $H^{0}(V ; \mathcal{O}) \otimes V^{*}$ of homogeneity 2 , namely the linear functions $g_{A}$ defined on the space $H^{0}\left(\Sigma ; K^{2}\right)$ of quadratic differentials. This space is canonically $H^{1}\left(\Sigma ; K^{-1}\right)$, so $\Psi$ defines an isomorphism between this space and the space $H^{1}\left(M^{s} ; T\right)$ which defines the classes in $H^{1}(\mathscr{M} ; \mathcal{O})$ of homogeneity 1 . To prove property (iv) we need to show that this is a non-zero multiple of the Kodaira-Spencer map. Now since $H^{1}\left(M^{s} ; \mathcal{O}\right)=0$, 
restriction of $H^{1}(\mathscr{M} ; \mathcal{O})$ to the first order neighbourhood of the zero-section $M^{s} \subset T^{*} M^{s}$ gives a map

$$
H^{1}(\mathscr{M} ; \mathcal{O}) \rightarrow H^{1}\left(M^{s} ; T\right) \cong H^{1}\left(M^{s} ; \mathcal{O}^{(1)}\right)
$$

which is the identity on $H^{1}\left(M^{s} ; T\right) \subset H^{1}(\mathscr{M} ; \mathcal{O})$ by the way we constructed this subspace.

Consider now the Hamiltonian vector field $X_{A}$ corresponding to the function $g_{A}$. In local coordinates $\left(z_{1}, \ldots, z_{n}\right)$ on $M^{s}$, suppose the canonical holomorphic symplectic form is $\sum_{i} d z_{i} \wedge d w_{i}$, then $g_{A}$ is the Hamiltonian function corresponding to a symmetric tensor

$$
G_{A}=\sum_{i, j} G_{A}^{i j} \frac{\partial}{\partial z_{i}} \otimes \frac{\partial}{\partial z_{j}}
$$

and so

$$
g_{A}(z, w)=\sum_{i, j} G_{A}^{i j} w_{i} w_{j}
$$

with Hamiltonian vector field

$$
X_{A}=-\sum_{i, j, k} \frac{\partial G_{A}^{i j}}{\partial z_{k}} w_{i} w_{j} \frac{\partial}{\partial w_{k}}+2 \sum G_{A}^{i j} w_{i} \frac{\partial}{\partial z_{j}} .
$$

Restricting to the first order neighbourhood of the zero section $w_{i}=0$ we just obtain the class

$$
2 \sum_{i, j, k} G_{A}^{i j} \omega_{j \bar{k}} \frac{\partial}{\partial z_{i}} \otimes d \bar{z}_{k},
$$

since the Kähler form restricts on $M^{s}$ to the standard Kähler form. This, from (2.16), is a non-zero multiple of the Kodaira-Spencer class.

The final property we require - the constancy of dimension of $H^{0}\left(M^{s} ; L^{k}\right)$ for the deformations of complex structure we are considering - now actually follows from the existence of a heat operator, which only requires properties (i)-(v). Evolving with the heat equation gives a canonical way of extending any section of $L^{k}$ on $M^{s}$ with one complex structure to nearby ones. This proves the required property without appealing to singular versions of Riemann-Roch and vanishing theorems in the style of Sect. 1.

Acknowledgements. The author wishes to thank M. F. Atiyah, G. B. Segal, and E. Witten for many enlightening discussions. Thanks are also due to the Royal Society and the European Community twinning programme SCI-0105-C(AM) (GADGET).

\section{References}

1. Atiyah, M.F.: Complex analytic connections in fibre bundles. Trans. Am. Math. Soc. 85, 181-207 (1957)

2. Atiyah, M.F., Bott, R.: The Yang-Mills equations over Riemann surfaces. Phil. Trans. R. Soc. Lond. A 308, 523-615 (1982)

3. Beauville, A., Narasimhan, M.S., Ramanan, S.: Spectral curves and the generalized theta divisor. J. Reine Angew. Math. 398, 169-179 (1989)

4. Griffiths, P., Harris, J.: Principles of algebraic geometry. New York: Wiley 1978 
5. Guillemin, V., Sternberg, S.: Geometric asymptotics. Mathematical surveys, Vol.14. Providence, RI: Am. Math. Soc., 1977

6. Hitchin, N.J.: The self-duality equations on a Riemann surface. Proc. Lond. Math. Soc. 55, 59-126 (1987)

7. Hitchin, N.J.: Stable bundles and integrable systems. Duke Math. J. 54, 91-114 (1987)

8. Kohno, T.: Monodromy representations of braid groups and Yang-Baxter equations. Ann. Inst. Fourier Grenoble 37, 139-160 (1987)

9. Knizhnik, V.G., Zamolodchikov, A.B.: Current algebra and Wess-Zumino model in two dimensions. Nucl. Phys. B 247, 83-103 (1984)

10. Nakamura, I.: On moduli of stable quasi-abelian varieties. Nagoya Math. J. 58, 149-214 (1975)

11. Narasimhan, M.S., Ramanan, S.: Deformations of the moduli space of vector bundles over an algebraic curve. Ann. Math. 101, 39-47 (1975)

12. Narasimhan, M.S., Seshadri, C.S.: Stable and unitary bundles on a compact Riemann surface. Ann. Math. 82, 540-564 (1965)

13. Nitsure, N.: Moduli space of semistable pairs on a curve. Proc. Lond. Math. Soc. (to appear)

14. Oda, T., Seshadri, C.S.: Compactification of the generalized Jacobian variety. Trans. Am. Math. Soc. 253, 1-90 (1979)

15. Quillen, D.: Determinants of Cauchy-Riemann operators over a Riemann surface. Funct. Anal. Appl. 19, 31-34 (1985)

16. Scheja, G.: Riemannsche Hebbarkeitsätze für Cohomologieklassen. Math. Ann. 144, 345-360 (1961)

17. Segal, G.B.: Conformal field theory. In: Proceedings of International Congress of Physics, Swansea 1988

18. Simpson, C.: Constructing variations of Hodge structure using Yang-Mills theory and applications to uniformization. J. Am. Math. Soc. 1, 867-918 (1988)

19. Tsuchiya, A., Ueno, K., Yamada, Y.: Conformal field theory on universal family of stable curves with gauge symmetries. Adv. Stud. Pure Math. 19 (1989): Integrable systems in quantum field theory and statistical mechanics, pp. 459-566

20. Welters, G.: Polarized abelian varieties and the heat equations. Compos. Math. 49, 173-194 (1983)

21. Witten, E.: Quantum field theory and the Jones polynomial. Commun. Math. Phys. 121, 351-399 (1989)

22. Witten, E., Axelrod, S., Della Pietra, S.: To appear

23. Woodhouse, N.: Geometric quantization. Oxford: Oxford University Press 1980

Communicated N. Yu. Reshetikhin

Received December 19, 1989 